\title{
Aspects of infinite permutation groups
}

\author{
Peter J. Cameron
}

School of Mathematical Sciences, Queen Mary, University of London, Mile End Road, London E1 4NS, UK

\begin{abstract}
Until 1980, there was no such subgroup as 'infinite permutation groups', according to the Mathematics Subject Classification: permutation groups were assumed to be finite. There were a few papers, for example $[10,62]$, and a set of lecture notes by Wielandt [72], from the 1950s.

Now, however, there are far more papers on the topic than can possibly be summarised in an article like this one.

I shall concentrate on a few topics, following the pattern of my conference lectures: the random graph (a case study); homogeneous relational structures (a powerful construction technique for interesting permutation groups); oligomorphic permutation groups (where the relations with other areas such as logic and combinatorics are clearest, and where a number of interesting enumerative questions arise); and the Urysohn space (another case study). I have preceded this with a short section introducing the language of permutation group theory, and I conclude with briefer accounts of a couple of topics that didn't make the cut for the lectures (maximal subgroups of the symmetric group, and Jordan groups).

I have highlighted a few specific open problems in the text. It will be clear that there are many wide areas needing investigation!
\end{abstract}

\section{Notation and terminology}

This section contains a few standard definitions concerning permutation groups. I write permutations on the right: that is, if $g$ is a permutation of a set $\Omega$, then the image of $\alpha$ under $g$ is written $\alpha g$.

The symmetric group $\operatorname{Sym}(\Omega)$ on a set $\Omega$ is the group consisting of all permutations of $\Omega$. If $\Omega$ is infinite and $c$ is an infinite cardinal number not exceeding $\Omega$, the bounded symmetric group $\operatorname{BSym}_{c}(\Omega)$ consists of all permutations moving fewer than $c$ points; if $c=\aleph_{0}$, this is the finitary symmetric group $\mathrm{FSym}(\Omega)$ consisting of all finitary permutations (moving only finitely many points). The alternating group $\operatorname{Alt}(\Omega)$ is the group of all even permutations, where a permutation is even if it moves only finitely many points and acts as an even permutation on its support.

Assuming the Axiom of Choice, the only nontrivial normal subgroups of $\operatorname{Sym}(\Omega)$ for an infinite set $\Omega$ are the bounded symmetric groups and the alternating group.

A permutation group on a set $\Omega$ is a subgroup of the symmetric group on $\Omega$. As noted above, we denote the image of $\alpha$ under the permutation $g$ by $\alpha g$. For the most part, I will be concerned with the case where $\Omega$ is countably infinite. 
The permutation group $G$ on $\Omega$ is said to be transitive if for any $\alpha, \beta \in \Omega$, there exists $g \in G$ with $\alpha g=\beta$. For $n \leq|\Omega|$, we say that $G$ is $n$-transitive if, in its induced action on the set of all $n$-tuples of distinct elements of $\Omega$, it is transitive: that is, given two $n$-tuples $\left(\alpha_{1}, \ldots, \alpha_{n}\right)$ and $\left(\beta_{1}, \ldots, \beta_{n}\right)$ of distinct elements, there exists $g \in G$ with $\alpha_{i} g=\beta_{i}$ for $i=1, \ldots, n$. In the case when $\Omega$ is infinite, we say that $G$ is highly transitive if if is $n$-transitive for all positive integers $n$. If a permutation group is not highly transitive, the maximum $n$ for which it is $n$ transitive is its degree of transitivity. (Of course, the condition of $n$-transitivity becomes stronger as $n$ increases.)

The bounded symmetric groups and the alternating group are all highly transitive. We will see that there are many other highly transitive groups!

The subgroup $G_{\alpha}=\{g \in G: \alpha g=\alpha\}$ of $G$ is the stabiliser of $\alpha$. Any transitive action of a group $G$ is isomorphic to the action on the set of right cosets of a point stabiliser, acting by right multiplication.

The permutation group $G$ on $\Omega$ is called semiregular (or free) if the stabiliser of any point of $\Omega$ is the trivial subgroup; and $G$ is regular if it is semiregular and transitive. Thus, a regular action of $G$ is isomorphic to the action on itself by right multiplication.

A transitive permutation group $G$ on $\Omega$ is imprimitive if there is a $G$-invariant equivalence relation on $\Omega$ which is not trivial (that is, not the relation of equality, and not the relation with a single equivalence class $\Omega$ ). If no such relation exists, then $G$ is primitive. A $G$-invariant equivalence relation is called a congruence; its equivalence classes are blocks of imprimitivity, and the set of blocks is a system of imprimitivity. A block or system of imprimitivity is non-trivial if the corresponding equivalence relation is. A non-empty subset $B$ of $\Omega$ is a block if and only if $B \cap B^{g}=$ $B$ or $\emptyset$ for all $g \in G$.

A couple of simple results about primitivity:

Proposition 1.1 (a) The transitive group $G$ on $\Omega$ is primitive if and only if $G_{\alpha}$ is a maximal proper subgroup of $G$ for some (or every) $\alpha \in \Omega$.

(b) A 2-transitive group is primitive.

(c) The orbits of a normal subgroup of a transitive group $G$ form a system of imprimitivity. Hence, a non-trivial normal subgroup of a transitive group is primitive.

In connection with the last part of this result, we say that a transitive permutation group is quasiprimitive if every non-trivial normal subgroup is transitive.

Let $G$ be a group, and $S$ a subset of $G$. The Cayley graph Cay $(G, S)$ is the (directed) graph with vertex set $G$, having directed edges $(g, s g)$ for all $g \in G$ and $s \in S$. If $1 \notin S$, then this graph has no loops; if $s \in S \Rightarrow s^{-1} \in S$, then it is an undirected graph (that is, whenever $(g, h)$ is an edge, then also $(h, g)$ is an edge, so we can regard edges as unordered pairs). It is easy to see that $\operatorname{Cay}(G, S)$ is connected if and only if $S$ generates $G$. Most importantly, $G$, acting on itself by right multiplication, is a group of automorphisms of $\operatorname{Cay}(G, S)$. In this situation, $G$ acts regularly on the vertex set of $\operatorname{Cay}(G, S)$. Conversely, if a graph $\Gamma$ admits 
a group $G$ as a group of automorphisms acting regularly on the vertices, then $\Gamma$ is isomorphic to a Cayley graph for $G$. (Choose a point $\alpha \in \Omega$, and take $S$ to be the set of elements $s$ for which $(\alpha, \alpha s)$ is an edge.)

\section{The random graph}

\section{$2.1 \quad$ B-groups}

According to Wielandt [71], a group $A$ is a $B$-group if any primitive permutation group $G$ which contains the group $A$ acting regularly is doubly transitive; that is, if any overgroup of $A$ which kills all the $A$-invariant equivalence relations necessarily kills all the non-trivial $A$-invariant binary relations. The letter $\mathrm{B}$ stands for Burnside, who showed that a cyclic group of prime-power but not prime order is a B-group. The proof contained a gap which was subsequently fixed by Schur, who invented and developed Schur rings for this purpose. The theory of Schur rings (or S-rings) is connected with many topics in representation theory, quasigroups, association schemes, and other areas of mathematics; historically, it was an important source of ideas in these subjects. The theory of S-rings and its connection with representation theory is described in Wielandt's book.

Following the classification of finite simple groups, much more is known about B-groups, since indeed it is known that primitive groups are comparatively rare. For example, the set of numbers $n$ for which there exists a primitive group of degree $n$ other than $S_{n}$ and $A_{n}$ has density zero [21], and hence the set of orders of non-B-groups has density zero. (However, there are non-B-groups of every prime power order, and a complete description is not known.)

One could then ask:

Are there any infinite B-groups?

Remarkably, no example of an infinite B-group is known. One of the most powerful nonexistence theorems is the following result. A square-root set in a group $X$ is a set of the form

$$
\sqrt{a}=\left\{x \in X: x^{2}=a\right\}
$$

it is non-principal if $a \neq 1$. A slightly weaker form of this theorem (using a stronger form of the condition, and concluding only that $A$ is not a B-group) was proved by Graham Higman; the form given here is due to Ken Johnson and me [20].

Theorem 2.1 Let $A$ be a countable group with the following property:

A cannot be written as the union of finitely many translates of nonprincipal square-root sets together with a finite set.

Then $A$ is not a B-group. More precisely, there exists a primitive but not 2transitive group $G$ which contains a regular subgroup isomorphic to each countable group satisfying this condition. 
Note that the condition of Theorem 2.1 is not very restrictive: any countable abelian group of infinite exponent satisfies the condition; and for any finite or countable group $G$, the direct product of $G$ with an infinite cyclic group satisfies it, so $G$ embeds every countable group as a semiregular subgroup.

\subsection{The Erdős-Rényi Theorem}

The group $G$ of the last subsection is the automorphism group of the remarkable random graph $R$ (sometimes known as the Rado graph). In the rest of this section we consider this graph and some of its properties.

The reason for the name is the following theorem of Erdős and Rényi [28]:

Theorem 2.2 There exists a countable (undirected simple) graph $R$ with the property that, if a graph $X$ on a fixed countable vertex set is chosen by selecting edges independently at random with probability $1 / 2$ from the unordered pairs of vertices, then $\operatorname{Prob}(X \cong R)=1$.

Proof The proof depends on the following graph property denoted by $(*)$ :

Given two finite disjoint sets $U, V$ of vertices, there exists a vertex $z$ joined to every vertex in $U$ and to no vertex in $V$.

Now the theorem is immediate from the following facts:

1. $\operatorname{Prob}(X$ satisfies $(*))=1$;

2 . Any two countable graphs satisfying $(*)$ are isomorphic.

To prove (1), we have to show that the event that $(*)$ fails is null. This event is the union of countably many events, one for each choice of the pair $(U, V)$ of sets; so it is enough to show that the probability that no $z$ exists for given $U, V$ is zero. But the probability that $n$ given vertices $z_{1}, \ldots, z_{n}$ fail to satisfy $(*)$, where $|U \cup V|=k$, is $\left(1-1 / 2^{k}\right)^{n}$, which tends to 0 as $n \rightarrow \infty$.

Claim (2) is proved by a simple back-and-forth argument. Any partial isomorphism between two countable graphs satisfying $(*)$ can be extended so that its domain or range contains one additional point. Proceeding back and forth for countably many steps, starting with the empty partial isomorphism, we obtain the desired isomorphism.

This is a fine example of a non-constructive existence proof: almost all countable graphs have property $(*)$, but no example of such a graph is exhibited! There are many constructions: here are a few. In each case, to show that the graph is isomorphic to $R$, we have to verify that $(*)$ holds.

1. Let $M$ be a countable model of the Zermelo-Fraenkel axioms of set theory. (The existence of such a model is Skolem's paradox.) Thus $M$ consists of a countable set carrying a binary relation $\in$. Form a graph by symmetrising this relation: that is, $\{x, y\}$ is an edge if $x \in y$ or $y \in x$. This graph is isomorphic to $R$. [Indeed, not all the ZF axioms are required here; it is enough to have the empty set, pair set, union, and foundation axioms.] 
2. As a specialisation of the above, we have Rado's model of hereditarily finite set theory (satisfying all the ZF axioms except the axiom of infinity): the set of elements is $\mathbb{N}$, and $x \in y$ if and only if the $x$ th digit in the base 2 expansion of $y$ is equal to 1 . This is the construction of $R$ in [54].

3. Let $\mathbb{P}_{1}$ be the set of primes congruent to $1 \bmod 4$; for $p, q \in \mathbb{P}_{1}$, join $p$ to $q$ if and only if $p$ is a quadratic residue mod $q$. (By quadratic reciprocity, this relation is symmetric.) Verification of $(*)$ uses Dirichlet's Theorem on primes in arithmetic progressions.

\subsection{Properties of $R$}

A graph $\Gamma$ is said to be homogeneous if every isomorphism between finite induced subgraphs extends to an automorphism of $\Gamma$.

Theorem 2.3 $R$ is homogeneous.

Proof Given an isomorphism between finite induced subgraphs, the back-and-forth method of the preceding proof extends it to an automorphism.

We now consider various properties of the group $G=\operatorname{Aut}(R)$. By homogeneity, $G$ acts transitively on the vertices, (oriented) edges, and (oriented) non-edges of $R$; so, acting on the vertex set, it is a transitive group with (permutation) rank 3 (that is, three orbits on ordered pairs of vertices). Moreover, $G$ is primitive on the vertices. For suppose that $\equiv$ is a congruence on the vertex set which is not the relation of equality, so that there are distinct vertices $v, v^{\prime}$ with $v \equiv v^{\prime}$. Suppose that $\left\{v, v^{\prime}\right\}$ is an edge (the argument in the other case is similar). Since $G$ is transitive on edges, it follows that for every edge $\left\{w, w^{\prime}\right\}$, we have $w \equiv w^{\prime}$. Now let $u, u^{\prime}$ be non-adjacent vertices. Choosing $U=\left\{u, u^{\prime}\right\}$ and $V=\emptyset$ in property (*), we find a vertex $z$ joined to $u$ and $u^{\prime}$. Thus $u \equiv z \equiv u^{\prime}$, so $u \equiv u^{\prime}$. Thus $\equiv$ is the universal congruence.

Thus, a group which can be embedded as a regular subgroup of $G$ (that is, a group $A$ for which some Cayley graph is isomorphic to $R$ ) is not a B-group. Now Theorem 2.1 is proved by showing that, if $A$ is a group satisfying the hypotheses of the theorem, then a random Cayley graph for $A$ is isomorphic to $R$ with probability 1, and hence that almost all Cayley graphs for $A$ are isomorphic to $R$.

As an example of the proof technique, I show:

Proposition 2.4 R has $2^{\aleph_{0}}$ non-conjugate cyclic automorphisms.

Proof If a graph $\Gamma$ has a cyclic automorphism $\sigma$, then we can index the vertices by integers so that $\sigma$ acts as the cyclic shift $x \mapsto x+1$. Then the graph $\Gamma$ is determined by the set $S=\{x \in \mathbb{Z}: x>0, x \sim 0\}$ : indeed, $\Gamma=\operatorname{Cay}(\mathbb{Z}, S \cup(-S))$. Furthermore, it is an easy calculation to show that, if the same graph $\Gamma$ has two cyclic automorphisms $\sigma_{1}$ and $\sigma_{2}$, giving rise to sets $S_{1}$ and $S_{2}$, then $S_{1}=S_{2}$ if and only if $\sigma_{1}$ and $\sigma_{2}$ are conjugate in $\operatorname{Aut}(\Gamma)$. So the theorem will be proved if we can show that there are $2^{\aleph_{0}}$ different sets $S$ for which the resulting graph $\Gamma$ is isomorphic to $R$. 
We do this by choosing the elements of $S$ independently at random from the positive integers, and showing that the probability that $(*)$ fails is zero. Suppose that we are testing, for a given pair $(U, V)$, whether there is a vertex $z$ "correctly joined". Of course, we must exclude the elements of $U \cup V$ from consideration. We must discard all $z$ for which we have already decided about the membership of any element of the form $|z-u|$ or $|z-v|$ in $S$, for $u \in U$ and $v \in V$ : there are finitely many such. We also discard any element $z$ for which an equation of the form $z-w_{1}=w_{2}-z$ holds, for $w_{1}, w_{2} \in U \cup V$; again there are only finitely many such $z$. So for all but finitely many $z$, the events $z \sim w$ for $w \in U \cup V$ are independent, and the probability that such a $z$ is correctly joined is non-zero.

Now the argument proceeds as in the proof of the Erdős-Rényi theorem. Since the event $\Gamma \cong R$ has probability 1 , it certainly has cardinality $2^{\aleph_{0}}$.

An example of a countable group for which no Cayley graph is isomorphic to $R$ is

$$
A=\left\langle a, b: b^{4}=1, b^{-1} a b=a^{-1}\right\rangle .
$$

Every element of the form $a^{i} b^{j}$ in this group with $j$ odd is a square root of $b^{2}$, and the remaining elements form a translate of this square root set. Hence, in a Cayley graph for $A$, we cannot find a point joined to 1 and $b$ but not to $b^{2}$ or $b^{3}$.

Problem 2.5 Is the above group $A$ a B-group?

\subsection{Properties of $\operatorname{Aut}(R)$}

A number of properties of the group $G=\operatorname{Aut}(R)$ are known:

(a) It has cardinality $2^{\aleph_{0}}$. (This follows from Proposition 2.4, or from (c) below.)

(b) It is simple [64].

(c) It has the strong small index property (see below) [34, 18].

Truss proved that $G$ is simple by showing that, given any two non-identity elements $g, h \in G$, it is possible to write $h$ as the product of five conjugates of $g^{ \pm 1}$. Subsequently [66] he improved this: only three conjugates of $g$ are required. This is best possible.

A permutation group on a countable set $X$ is said to have the small index property if every subgroup of index less than $2^{\aleph_{0}}$ contains the pointwise stabiliser of a finite set; it has the strong small index property if every such subgroup lies between the pointwise and setwise stabilisers of a finite set. Hodges et al. [34] showed that $G$ has the small index property, and Cameron [18] improved this to the strong small index property.

Truss [65] found all the cycle structures of automorphisms of $R$. In particular, $R$ has cyclic automorphisms. (This is the assertion that the infinite cyclic group is a regular subgroup of $G$.)

A number of subgroups of $G$ with remarkable properties have been shown to exist. Here are two examples, due to Bhattacharjee and Macpherson [6, 7]. The first settles a question of Peter Neumann. 
Theorem 2.6 There exist automorphisms $f, g$ of $R$ such that

(a) $f$ has a single cycle on $R$, which is infinite,

(b) $g$ fixes a vertex $v$ and has two cycles on the remaining vertices (namely, the neighbours and non-neighbours of $v$ ),

(c) the group $\langle f, g\rangle$ is free and is transitive on vertices, edges, and non-edges of $R$, and each of its non-identity elements has only finitely many cycles on $R$.

This theorem is proved by building the permutations $f$ and $g$ as limits of partial maps constructed in stages. Of course the existence of automorphisms satisfying (a) and (b) follows from Truss' classification of cycle types, but much more work is required to achieve (c).

Theorem 2.7 There is a locally finite group $G$ of automorphisms of $R$ which acts homogeneously (that is, any isomorphism between finite subgraphs can be extended to an element of $G$ ).

This theorem uses a result of Hrushovski [35] on extending partial automorphisms of graphs.

Various other subgroups acting homogeneously on $R$ can be constructed. For example, using either of the explicit descriptions of $R$ given earlier, the group of automorphisms given by recursive (or primitive recursive) functions (on $\mathbb{N}$ or $\mathbb{P}_{1}$ respectively) acts homogeneously.

We noted that $\operatorname{Aut}(R)$ embeds all finite or countable groups. On a similar note, Bonato et al. [8] showed that the endomorphism monoid of $R$ embeds all finite or countable monoids.

\subsection{Topology}

I now turn to properties of $G$ as a topological group. There is a natural topology on the symmetric group of countable degree, the topology of pointwise convergence: a basis of neighbourhoods of the identity is given by the stabilizers of finite tuples. (The intuition is that two permutations are close together if they agree on a large finite subset of the domain.) This topology is derived from either of the following two metrics. (We identify the permutation domain with $\mathbb{N}$.)

(a) $d(g, g)=0$ and $d(g, h)=1 / 2^{n}$ where $n$ is such that $i g=i h$ for $i<n$ but $n g \neq n h$, for $g \neq h$.

(b) $d^{\prime}(g, g)=0$ and $d^{\prime}(g, h)=1 / 2^{n}$ where $n$ is such that $i g=i h$ and $i g^{-1}=i h^{-1}$ for $i<n$ but either $n g \neq n h$ or $n g^{-1} \neq n h^{-1}$, for $g \neq h$.

(Here $1 / 2^{n}$ could be replaced by any decreasing sequence tending to zero.) The advantage of the metric $d^{\prime}$ is that the symmetric group is a complete metric space for this metric. (The cycles $(1,2, \ldots, n)$ form a Cauchy sequence for $d$ which does not converge to a permutation.)

The closed and open subgroups of the symmetric group can be characterised as follows. A first-order structure consists of a set carrying a collection of relations and functions (of various positive arities) and constants; it is relational if there are no 
functions or constants. Thus, a graph is an example of a relational structure (with a single binary relation). Just as for graphs, a relational structure $M$ is homogeneous if any isomorphism between finite induced substructures can be extended to an automorphism of $M$.

Theorem 2.8 (a) A subgroup $H$ of the symmetric group is open if and only if it contains the stabiliser of a finite tuple.

(b) A subgroup $H$ of the symmetric group is closed if and only if it is the full automorphism group of some first-order structure $M$; this structure can be taken to be a homogeneous relational structure.

The first part of this theorem gives an interpretation of the small index property:

Proposition 2.9 A closed subgroup $G$ of the symmetric group of countable degree has the small index property if and only if any subgroup of $G$ with index less than $2^{\aleph_{0}}$ is open in $G$.

Thus, if a group has the small index property, then its topology can be recovered from its group structure: the subgroups of index less than $2^{\aleph_{0}}$ form a basis of open neighbourhoods of the identity.

For the second part of the theorem, we define the canonical relational structure associated with a permutation group $G$ as follows: For each orbit $O$ of $G$ on $n$-tuples of points of the domain $\Omega$, we take an $n$-ary relation $R_{O}$, and specify that $R_{O}$ holds precisely for those $n$-tuples which belong to the orbit $O$. The resulting structure is easily seen to be homogeneous. Now the closure of $G$ is the automorphism group of its canonical relational structure.

A subgroup $H$ of $G$ is dense in $G$ if and only if $G$ and $H$ have the same orbits on $\Omega^{n}$ for all $n \in \mathbb{N}$. In particular, a subgroup $H$ of $\operatorname{Aut}(R)$ is dense in $\operatorname{Aut}(R)$ if and only if it acts homogeneously on $R$ (as described earlier). Thus, for example, the second theorem of Bhattacharjee and Macpherson asserts that $\operatorname{Aut}(R)$ has a locally finite dense subgroup.

A remarkable classification of closed subgroups of the symmetric group of countable degree has been given by Bergman and Shelah [5]. Call two subgroups $G_{1}$ and $G_{2}$ of $\operatorname{Sym}(\Omega)$ equivalent if there exists a finite subset $U$ of $\operatorname{Sym}(\Omega)$ such that $\left\langle G_{1} \cup U\right\rangle=\left\langle G_{2} \cup U\right\rangle$.

Theorem 2.10 If $\Omega$ is countable, there are just four equivalence classes of closed subgroups of $\operatorname{Sym}(\Omega)$. They are characterised by the following conditions, where $G_{(\Gamma)}$ denotes the pointwise stabiliser of the subset $\Gamma$ :

(a) For every finite subset $\Gamma$ of $\Omega$, the subgroup $G_{(\Gamma)}$ has at least one infinite orbit on $\Omega$.

(b) There exists a finite subset $\Gamma$ such that all the orbits of $G_{(\Gamma)}$ are finite, but none such that the cardinalities of these orbits have a common upper bound.

(c) There exists a finite subset $\Gamma$ such that the orbits of $G_{(\Gamma)}$ have a common upper bound, but none such that $G_{(\Gamma)}=1$. 
(d) There exists a finite subset $\Gamma$ such that $G_{(\Gamma)}=1$.

Note that $\operatorname{Aut}(R)$ falls into class (a) of this theorem; in fact, for any finite set $\Gamma$, all the orbits of $G_{(\Gamma)}$ outside $\Gamma$ are infinite, and have the property that the induced subgraph is isomorphic to $R$ and $G_{(\Gamma)}$ acts homogeneously on it. (If $|W|=n$, then the pointwise stabiliser of $W$ has $2^{n}$ orbits outside $W$ : for each subset $U$ of $W$, there is an orbit consisting of the points witnessing condition $(*)$ for the pair $(U, V)$, where $V=W \backslash U$.) So there is a finite set $B$ such that $\langle\operatorname{Aut}(R), B\rangle=\operatorname{Sym}(R)$. (This fact follows from Theorem 1.1 of [43]. Galvin [30] showed that we can take $B$ to consist of a single element. As we will see in the final section of the paper, these results are not specific to $\operatorname{Aut}(R)$.)

Recall that a subset of a metric space is residual if it contains a countable intersection of dense open sets. The Baire category theorem states that a residual set in a complete metric space is non-empty. Indeed, residual sets are 'large'; their properties are analogous to those of sets of full measure in a measure space. (For example, the intersection of countably many residual sets is dense.)

Now let $G$ denote any closed subgroup of the symmetric group of countable degree. The element $g \in G$ is said to be generic if its conjugacy class is residual in $G$. A given group has at most one conjugacy class of generic elements. The first part of the following theorem is due to Truss [65]; the second is due to Hodges et al. [34], and is crucial for proving the small index property for $\operatorname{Aut}(R)$.

Theorem 2.11 (a) The group $\operatorname{Aut}(R)$ contains generic elements. Such an element has infinitely many cycles of any given finite length and no infinite cycles.

(b) For any positive integer $n$, the group $\operatorname{Aut}(R)^{n}$ contains generic elements. Such an n-tuple generates a free subgroup of $\operatorname{Aut}(R)$, all of whose orbits are finite.

\section{$2.6 \quad$ Reducts of $R$}

We conclude this section with Thomas' theorem [60] about the reducts of $R$. For my purposes here, a reduct of a structure $A$ is a structure $B$ on the same set with $\operatorname{Aut}(B) \geq \operatorname{Aut}(A)$. If our structures are first-order, then their automorphism groups are closed subgroups of the symmetric group on $A$; so we are looking for closed overgroups of $\operatorname{Aut}(A)$.

An anti-automorphism of a graph $\Gamma$ is a permutation of the vertices mapping $\Gamma$ to its complement. A switching automorphism is a permutation mapping $\Gamma$ to a graph equivalent to it under switching. (Here switching with respect to a set $X$ of vertices consists in replacing edges between $X$ and its complement by nonedges, and non-edges by edges, leaving edges within or outside $X$ unchanged.) A switching anti-automorphism is a permutation mapping $\Gamma$ to a graph equivalent to its complement under switching. Now Thomas' Theorem asserts:

Theorem 2.12 The closed subgroups of $\operatorname{Sym}(R)$ containing $\operatorname{Aut}(R)$ are:

- $\operatorname{Aut}(R)$; 
- the group of automorphisms and anti-automorphisms of $R$;

- the group of switching automorphisms of $R$;

- the group of switching automorphisms and anti-automorphisms of $R$;

- $\operatorname{Sym}(R)$.

\section{$3 \quad$ Homogeneous relational structures}

\subsection{Fraïssé's Theorem}

For finite permutation groups, as is well known, a consequence of the Classification of Finite Simple Groups is the fact that the only finite 6-transitive permutation groups are the symmetric and alternating groups. What about the infinite case? Analogues of the "geometric" multiply-transitive finite permutation groups such as projective general linear groups give groups which are at most 3-transitive. Can we achieve higher degrees of transitivity?

I do not know any trivial construction which produces an infinite permutation group with any prescribed degree of transitivity. If we take $\Omega$ to be countable, and let $g_{i}$ be any permutation fixing $i$ points and permuting the others in a single cycle, then it is clear that $\left\langle g_{0}, \ldots, g_{n-1}\right\rangle$ is $n$-transitive; but it may be $(n+1)$-transitive, or even highly transitive. A sufficiently clever choice of the permutations might give a group which is not $(n+1)$-transitive. However, it is more straightforward to ensure that the group is not $(n+1)$-transitive by letting it preserve a suitable $(n+1)$-ary relation, and to choose this relation to have an $n$-transitive symmetry group.

A theorem of Fraïssé guarantees the existence of suitable structures. I now describe this theorem.

We work in the context of relational structures over a fixed relational language (that is, the relations are named, and relations with the same name in different structures have different names; moreover, the induced substructure on a subset of a relational structure is obtained by restricting all relations to the subset). Fraïssé defined the age $\operatorname{Age}(M)$ of a relational structure $M$ to be the class of all finite relational structures embeddable (as induced substructure) in $M$. Thus, the age of the random graph $R$ consists of all finite graphs.

As noted earlier, a relational structure is homogeneous if every isomorphism between finite substructures extends to an automorphism. Thus, the random graph $R$ is homogeneous. Another homogeneous relational structure, which was Fraïssé's motivating example, is the set $\mathbb{Q}$ of rational numbers as ordered set. (It is easy to see that any order-preserving map between two $n$-tuples of rationals can be extended to a piecewise-linear order-preserving map on the whole of $\mathbb{Q}$.)

The following straightforward result allows us to recognise ages. The joint embedding property of a class $\mathcal{C}$ asserts that, for all $B_{1}, B_{2} \in \mathcal{C}$, there exists $C \in \mathcal{C}$ such that both $B_{1}$ and $B_{2}$ can be embedded in $C$.

Proposition 3.1 Let $\mathcal{C}$ be a class of finite relational structures over a fixed relational language. Then $\mathcal{C}$ is the age of a countable relational structure $M$ if and 
only if the following conditions hold:

(a) $\mathcal{C}$ is closed under isomorphism;

(b) $\mathcal{C}$ is closed under taking induced substructures;

(c) $\mathcal{C}$ contains only finitely many structures up to isomorphism;

(d) $\mathcal{C}$ has the joint embedding property.

An age $\mathcal{C}=\operatorname{Age}(M)$ can be represented by a rooted tree: the nodes on level $n$ are the structures in $\mathcal{C}$ on the set $\{0,1, \ldots, n-1\}$, and the children of a structure are all its one-point extensions. Now the elements of the boundary of $\mathcal{C}$ (the infinite paths starting at the root) represent, in a natural way, structures $N$ on the set $\mathbb{N}$ satisfying $\operatorname{Age}(N) \subseteq \operatorname{Age}(M)$. (Fraïssé calls such structures $N$ younger than $M$.) I note in passing that the set of such paths has a natural hypermetric (the distance between two paths being a decreasing function of the number of steps for which they coincide), and is complete in this metric; so we can talk about residual properties of structures younger than $M$. A simple example: of the structures younger than $M$, a residual set have the same age as $M$.

Fraïssé [29] gave a necessary and sufficient condition for a class $\mathcal{C}$ of finite structures to be the age of a homogeneous relational structure. This involves the amalgamation property, the assertion that if $A, B_{1}, B_{2} \in \mathcal{C}$ and $f_{i}: A \rightarrow B_{i}$ are embeddings for $i=1,2$, then there is a structure $C \in \mathcal{C}$ and embeddings $g_{i}: B_{i} \rightarrow C$ for $i=1,2$, forming a commutative diagram (that is, $f_{1} g_{1}=f_{2} g_{2}$ ). Note that I allow the empty structure as a substructure; hence the amalgamation property as stated here includes the joint embedding property. (This depends on the fact that there is only one empty set, which holds because we do not allow relations of arity 0.) Note also that $A f_{1} g_{1} \subseteq B_{1} g_{1} \cap B_{2} g_{2}$; but it is not required that the images of $B_{1}$ and $B_{2}$ in $C$ intersect just in the image of $A$. (Later we say that the strong amalgamation property holds if this condition can be required.)

Theorem 3.2 Let $\mathcal{C}$ be a class of finite relational structures over a fixed relational language. Then $\mathcal{C}$ is the age of a countable homogeneous relational structure $M$ if and only if the following conditions hold:

(a) $\mathcal{C}$ is closed under isomorphism;

(b) $\mathcal{C}$ is closed under taking induced substructures;

(c) $\mathcal{C}$ contains only finitely many structures up to isomorphism;

(d) $\mathcal{C}$ has the amalgamation property.

If the conditions (a)-(d) hold, then the countable homogeneous structure $M$ is unique up to isomorphism.

A class $\mathcal{C}$ satisfying (a)-(d) of the theorem is called a Fraïssé class, and the unique countable homogeneous structure $M$ with age $\mathcal{C}$ is its Fraïssé limit. For example, the classes of finite graphs and finite linearly ordered sets are Fraïssé classes; their Fraïssé limits are respectively the random graph and the ordered set of rationals.

A more general form of Fraïssé's Theorem has been found by Hrushovski, who has used it to produce some remarkable examples in model theory (such as a coun- 
terexample to Lachlan's pseudoplane conjecture). These structures have interesting automorphism groups which have not been much investigated in their own right.

Hrushovski's method relies on the fact that we do not have to require all instances of the amalgamation property in the class $\mathcal{C}$, but only those where $A$ is "closed" in a suitable sense. I will not attempt further explanation here; there is a good exposition of this theory by Wagner [70].

\subsection{Applications}

As a first application of Fraïssé's Theorem, I show that groups with any desired degree of transitivity exist. Let $\mathcal{C}_{n}$ be the class of $(n+1)$-uniform hypergraphs: these are structures whose edges are sets of $n+1$ points, rather than two points as in the case of graphs. (As relational structures, we have one $(n+1)$-ary relation, which holds only if its arguments are all distinct and is completely symmetric.) It is easy to see that $\mathcal{C}_{n}$ is a Fraïssé class; let $R_{n}$ be its Fraïssé limit, and $G_{n}=\operatorname{Aut}\left(R_{n}\right)$. Then

(a) $G_{n}$ is $n$-transitive. This is because, by definition, the induced substructure on any $n$-set is trivial (with no edges), and so any bijection between $n$-sets is an isomorphism of induced substructures; by homogeneity, such an isomorphism extends to an automorphism of $R_{n}$.

(b) $G_{n}$ is not $(n+1)$-transitive. This is because no automorphism can map $n+1$ points forming an edge to $n+1$ points not forming an edge.

Of course, $R_{1}$ is the random graph $R$. In a similar way, $R_{n}$ is the random $(n+1)$ uniform hypergraph. It has a number of properties which resemble or extend those of $R$. Here are a few:

(a) Every countable group can be embedded as a regular subgroup of $R_{n}$ if $n>1$.

(b) All reducts of $R_{n}$ are known (Thomas [61]).

(c) There are explicit constructions of $R_{n}$.

In fact, the hypergraphs $R_{n}$ were constructed by Rado in the same paper in which he considered the graph $R$. I outline the construction. There is a nice bijection $F_{n}$ from the set of $n$-element subsets of $\mathbb{N}$ to the set $\mathbb{N}$, defined as follows: if $x_{0}<x_{1}<\cdots<x_{n-1}$, then

$$
F_{n}\left(\left\{x_{0}, x_{1}, \ldots, x_{n-1}\right\}\right)=\left(\begin{array}{c}
x_{0} \\
1
\end{array}\right)+\left(\begin{array}{c}
x_{1} \\
2
\end{array}\right)+\cdots+\left(\begin{array}{c}
x_{n-1} \\
n
\end{array}\right) .
$$

Now, for $x_{0}<\cdots<x_{n-1}<y$, we take $\left\{x_{0}, x_{1}, \ldots, x_{n-1}, y\right\}$ to be an edge of $R_{n}$ if and only if the $m$ th digit in the base 2 expansion of $y$ is equal to 1 , where $m=F_{n}\left(\left\{x_{0}, x_{1}, \ldots, x_{n-1}\right\}\right)$. This reduces to Rado's construction of $R$ when $n=1$.

Further interesting groups can be obtained by exploiting also a method due to Tits [63]. (He first used this to show that the free group of countable rank has BN-pairs of all spherical types.)

Theorem 3.3 Suppose that $G$ is a permutation group on a countable set $\Omega$, and $N$ a normal subgroup of $G$ having the same orbits as $G$. Suppose that $G / N$ contains 
a non-abelian free subgroup. Then $G$ has a subgroup $F$, which is a free group of countable rank, having the same orbits on $\Omega$ as $G$ and satisfying $F \cap N=1$.

Proof Let $f_{1} N, f_{2} N, \ldots$ be free generators of a free subgroup of countable rank in $G / N$. Enumerate all pairs $\left(\alpha_{i}, \beta_{i}\right)$ of points for which $\alpha_{i}$ and $\beta_{i}$ lie in the same $G$-orbit on $\Omega$, and choose elements $n_{i} \in N$ such that $\alpha_{i} f_{i} n_{i}=\beta_{i}$. Then it is easy to show that $F=\left\langle f_{1} n_{1}, f_{2} n_{2}, \ldots\right\rangle$ is the required subgroup.

The first application, due to Kantor, shows that $F$ has a faithful highly transitive action. We take $\Omega$ to be the union, over all $n$, of the sets of $n$-tuples of distinct elements of a fixed countable set $X$; let $G$ be the symmetric group on $X$, and $N$ the finitary symmetric group consisting of permutations of finite support. Then a subgroup of $G$ has the same orbits on $\Omega$ as $G$ if and only if it is highly transitive on $X$. Embed $F$ into $G$ by means of its regular representation; then $F \cap N=1$, so $F$ embeds into $G / N$. The conditions of the theorem are easily checked.

This action of $F$ has the further property that it is cofinitary, that is, any nonidentity element fixes only finitely many points. For any non-identity element of the subgroup $F$ is the product of a non-identity element of the regular copy of $F$ and a finitary permutation.

The application of the theorem is limited by the requirement that $G$ should have a normal subgroup which is 'not too big and not too small'. For example, since $\operatorname{Aut}(R)$ is simple, we cannot apply this directly to $R$. Often it is possible to refine the structure with extra relations. Here is an example.

Theorem 3.4 For every positive integer $k$, there is a permutation group which is $k$-transitive, in which the stabiliser of any $k+1$ points is the identity.

This theorem is best possible, since another theorem due to Tits [62] and Hall [31] independently shows that, for $k \geq 4$, there is no sharply $k$-transitive group, that is no $k$-transitive group in which the stabiliser of any $k$ points is the identity. The groups of the theorem are "higher analogues" of Frobenius and Zassenhaus groups.

Proof Take the class $\mathcal{C}$ of finite structures each of which consists of a colouring of the $(k+1)$-subsets of its domain with a fixed set of countably many colours. Then $\mathcal{C}$ is a Fraïssé class; let $M$ be its Fraïssé limit. Let $G$ be the group of automorphisms of $M$ (where automorphisms are allowed to permute the colours), and $N$ the subgroup fixing all the colours. Then $G / N$ is the symmetric group on the set of colours, and certainly contains a free group of countable rank acting regularly. Moreover, both $G$ and $N$ are $k$-transitive. Applying the theorem, we find a $k$-transitive copy of $F$ inside $G$. The construction shows that $F$ acts regularly on the set of colours; so the stabiliser of any $(k+1)$-set, and a fortiori any $(k+1)$-tuple, is trivial.

Other strange permutation groups can be constructed with 'bare hands', where permutations generating the required group are built up in stages. Such methods require a great deal of ingenuity. Many beautiful examples have been given by Macpherson. We have already seen two of his theorems with Bhattacharjee [6, 7]; here is another result [42]. 
Theorem 3.5 For any $k \geq 1$, the group of order-automorphisms of the rational numbers contains a free subgroup $F$ of countable rank with the properties that for any two $k$-tuples $x_{1}<x_{2}<\cdots<x_{k}$ and $y_{1}<y_{2}<\cdots<y_{k}$, there is a unique element of $F$ mapping the first to the second. (In other words, $F$ is "sharply $k$-set transitive".)

This result answered an open question on scale type in the theory of measurement, an area with close links with various aspects of permutation group theory.

Macpherson also showed that $\operatorname{Aut}\left(R_{n}\right)$ contains an automorphism fixing $i$ points and acting as a single cycle on the rest, for $0 \leq i \leq n-1$. As we saw at the start of this section, the group generated by such permutations is obviously $n$-transitive; in this case it cannot be $(n+1)$-transitive, since it leaves the hypergraph $R_{n}$ invariant. So, at least in principle, the construction of groups with any degree of transitivity hinted at there can be made to work. Macpherson further showed that we can take the group generated by these automorphisms to be free.

\subsection{Homogeneous structures}

A lot of effort has gone into classifying the homogeneous structures of various types. One important result is due to Lachlan and Woodrow [39]:

Theorem 3.6 A countable homogeneous (undirected) graph is one of the following:

(a) the disjoint union of $m$ complete graphs of size $n$, where $m n=\aleph_{0}$;

(b) complements of (a);

(c) the Fraissé limit of the class of graphs containing no complete subgraph of size $n$, for $n \geq 3$;

(d) complements of (c);

(e) the random graph $R$.

Other classes in which the homogeneous structures have been determined include directed graphs (Cherlin [25], the case of tournaments having been done earlier by Lachlan [38]) and posets (Schmerl [57]).

The Fraïssé limit of the class of $K_{n}$-free graphs is known as the Henson graph $H_{n}$, since it was first constructed by Henson [32]. One of Henson's results was that $H_{3}$ admits cyclic automorphisms but $H_{n}$ does not if $n>3$. This can be extended as follows.

The triangle-free graph $H_{3}$ has the property that it admits many regular subgroups, and so is a Cayley graph for many different groups. The proof is similar to that for $R$. However, for $n>3$, it can be shown that $H_{n}$ is not a normal Cayley graph for any countable group (where a Cayley graph $\operatorname{Cay}(G, S)$ is normal if it is invariant under both left and right translation by $G$, or in other words, if $S$ is a normal subset of $G$ ). In particular, it is not a Cayley graph of any countable abelian group.

Problem 3.7 Is $H_{n}$ a Cayley graph for $n>3$ ? 
The graph $H_{3}$ also gives us another highly transitive group, namely the stabiliser of a vertex $v$ in $\operatorname{Aut}\left(H_{3}\right)$, acting on the set of neighbours of $v$.

\subsection{Some recent connections}

I conclude this section with a brief account of two recent occurrences of homogeneous structures and Fraïssé classes in very different parts of mathematics: Ramsey theory and topological dynamics.

I begin with a simple form of the finite version of Ramsey's Theorem.

Theorem 3.8 For any two natural numbers $a, b$ with $a<b$, there exists a natural number $c$ with $b<c$ having the property that, if the a-element subsets of a c-element set are coloured red and blue in any manner, then there exists a b-element subset, all of whose a-element subsets have the same colour.

In order to convert this theorem into a theory, we might take a fixed type of relational structure, replace "set" and "subset" by "structure" and "substructure", and ask whether the analogue of Ramsey's Theorem holds. To formalise this, let $\mathcal{C}$ be a class of finite relational structures. For $A, B \in \mathcal{C}$, we denote by $\left(\begin{array}{l}B \\ A\end{array}\right)$ the class of all substructures of $B$ isomorphic to $A$. We say that $\mathcal{C}$ is a Ramsey class if the following holds:

For any $A, B \in \mathcal{C}$ with $A$ embeddable in $B$, there exists $C \in \mathcal{C}$ with $B$ embeddable in $C$ such that, if the elements of $\left(\begin{array}{l}C \\ A\end{array}\right)$ are coloured red and blue in any manner, there exists $B^{\prime} \in\left(\begin{array}{l}C \\ B\end{array}\right)$ such that all elements of $\left(\begin{array}{c}B^{\prime} \\ A\end{array}\right)$ have the same colour.

It is natural to assume that $\mathcal{C}$ is isomorphism-closed and substructure-closed. Usually we assume that it is an age.

Various deep theorems assert that the classes of graphs and of triangle-free graphs are Ramsey classes. Taking this hint, Nešetřil [47] showed the following result:

Theorem 3.9 Let $\mathcal{C}$ be the age of a countable structure (that is, let it satisfy conditions (a)-(d) of Proposition 3.1). If $\mathcal{C}$ is a Ramsey class, then it is a Fraïssé class (that is, it satisfies conditions (a)-(d) of Theorem 3.2).

Proof Suppose that $\mathcal{C}$ is a Ramsey class but not a Fraïssé class. Then the amalgamation property fails in $\mathcal{C}$; that is, there exist embeddings $f_{1}: A \rightarrow B_{1}, f_{2}: A \rightarrow B_{2}$ which cannot be amalgamated. However, $\mathcal{C}$ is an age, so we can find $C \in \mathcal{C}$ into which $B_{1}$ and $B_{2}$ can be embedded. (Without loss, we assume that $B_{1}$ and $B_{2}$ are substructures of $C$.) Now by the Ramsey property, we can find $D$ such that, if the elements of $\left(\begin{array}{c}D \\ A\end{array}\right)$ are coloured red and blue in any manner, there is a monochromatic copy $C^{\prime}$ of $C$.

Colour the elements of $\left(\begin{array}{l}D \\ A\end{array}\right)$ as follows: the structure $A^{\prime} \cong A$ is red if it is contained in a structure $B^{\prime} \cong B_{1}$ inside $D$; otherwise it is blue. Now find $C^{\prime} \cong C$ inside $D$ such that all copies of $A$ in $C^{\prime}$ have the same colour. This colour must be 
red, since we know that $C$ contains at least one copy of $A$ which is inside a copy of $B_{1}$ (within $C$ ), namely $A f_{1}$. So all copies of $A$ in $C^{\prime}$ are red. But we know there is a copy of $B_{2}$ in $C$; so the image $A f_{2}$ of $A$ under its embedding in $B_{2}$ is contained in $B^{\prime} \cong B_{1}$ (within $D$ ). Then $B^{\prime} \cup B_{2}$ is an amalgam, contrary to assumption.

This suggests a programme for determining the Ramsey classes of some given type. (It is reasonable to look only for classes which are ages.)

- Find the Fraïssé classes of that type.

- Decide which of them are Ramsey classes.

Before the last topic, we make a small digression. Some relational structures naturally carry a total order (for example, those for which one of the relations is interpreted as a total order in the structure). Often, if there is not a natural order on the structure, we can add one.

We say that a Fraïssé class $\mathcal{C}$ has the strong amalgamation property if, in the statement of the a.p., we may assume that $B_{1} g_{1} \cap B_{2} g_{2}=A f_{1} g_{1}$, that is, the intersection of the images of $B_{1}$ and $B_{2}$ in the amalgam is no larger than it has to be. It can be shown that, if $\mathcal{C}$ is a Fraïssé class with Fraïssé limit $M$, then $\mathcal{C}$ has the strong amalgamation property if and only if $G=\operatorname{Aut}(M)$ has the property that the stabiliser of finitely many points in $G$ has no finite orbits on the remaining points.

If $\mathcal{C}_{1}$ and $\mathcal{C}_{2}$ are Fraïssé classes with the strong a.p., then we can form a class $\mathcal{C}_{1} \wedge \mathcal{C}_{2}$ whose members consist of a $\mathcal{C}_{1}$-structure and a $\mathcal{C}_{2}$-structure on the same set; we amalgamate the two types of structure independently. In particular, since the class of total orders has the strong a.p., we see that if $\mathcal{C}$ has the strong a.p., then there is a class $\mathcal{C}^{<}$whose elements are totally ordered versions of $\mathcal{C}$-structures. So, in this case, if we do not have an order already, we can impose one.

Now we can discuss the result of Kechris, Pestov, and Todorcevic [37]. In this section, all topological spaces and groups will be assumed to be Hausdorff.

A topological group $G$ is extremely amenable if, whenever $G$ acts continuously on a compact space, it has a fixed point.

This condition is stronger than the usual forms of amenability which have been studied, which merely require the existence of an invariant measure in this situation. Indeed, a theorem of Veech [68] shows that no locally compact group can be extremely amenable, since such a group always has a free action on a compact space. However, extremely amenable groups do exist. The remarkable theorem of Kechris et al. asserts the following. Here a closed subgroup of the symmetric group $\operatorname{Sym}(\Omega)$ has the topology of pointwise convergence (the induced topology from $\operatorname{Sym}(\Omega)$ ). Recall that any closed subgroup of $\operatorname{Sym}(\Omega)$, for countable $\Omega$, is the automorphism group of some homogeneous relational structure $M$ on $\Omega$.

Theorem 3.10 Let $G$ be the automorphism group of a countable homogeneous relational structure $M$. Then the following are equivalent:

(a) $G$ is extremely amenable;

(b) the structure on $M$ includes a total order, and $\operatorname{Age}(M)$ is a Ramsey class. 
In particular, $\operatorname{Aut}(\mathbb{Q},<)$ is extremely amenable, as are $\operatorname{Aut}\left(R^{<}\right)$and $\operatorname{Aut}\left(H_{n}^{<}\right)$ for $n \geq 3$, where $H_{n}$ is Henson's graph.

Even in cases where the structure on $M$ does not include a total order, the methods of the paper allow the possible minimal $G$-flows (minimal continuous actions on compact spaces) to be determined. However, I cannot tell more of this fascinating story here!

\section{Oligomorphic permutation groups}

\subsection{Definition and basic properties}

Let $G$ be a permutation group on a set $\Omega$. (Usually we assume that $\Omega$ is infinite.) We say that $G$ is oligomorphic if it has only finitely many orbits on $\Omega^{n}$ for all natural numbers $n$. (By convention, $\Omega^{0}$ is a single point.)

There are three convenient numbers with the property that, if one is finite (for some value of $n$ ) then so are the others:

$F_{n}^{*}$ : the number of $G$-orbits on $\Omega^{n}$;

$F_{n}$ : the number of $G$-orbits on $n$-tuples of distinct elements of $\Omega$;

$f_{n}$ : the number of $G$-orbits on $n$-element subsets of $\Omega$.

If we need to specify the group under consideration, we write $F_{n}^{*}(G)$, etc.

The first two sequences determine each other, since

$$
F_{n}^{*}=\sum_{k=1}^{n} S(n, k) F_{k}
$$

for $n \geq 1$, where $S(n, k)$ is the Stirling number of the second kind, the number of partitions of an $n$-set into $k$ parts. Because of this, we concentrate on the sequence $F_{n}$. The permutation group $G$ is highly transitive if and only if $F_{n}(G)=1$ for all $n \in \mathbb{N}$. If this holds, then $F_{n}^{*}(G)=B(n)$, the $n$th Bell number (the number of partitions of an $n$-set).

The second and third sequences clearly satisfy

$$
f_{n} \leq F_{n} \leq n ! f_{n},
$$

but neither determines the other in general.

Let $G$ be oligomorphic. We denote by $F^{*}(x)$ and $F(x)$ the exponential generating functions

$$
F^{*}(x)=\sum_{n \geq 0} \frac{F_{n}^{*} x^{n}}{n !}, \quad F(x)=\sum_{n \geq 0} \frac{F_{n} x^{n}}{n !} .
$$

It follows from our observation about the relation between these sequences that $F^{*}(x)=F\left(\mathrm{e}^{x}-1\right)$.

Let $f(x)$ be the ordinary generating function

$$
f(x)=\sum_{n \geq 0} f_{n} x^{n} .
$$


All of these are specialisations of a multi-variable power series which we call the modified cycle index of $G$, defined by

$$
\tilde{Z}(G)=\sum_{A \in \mathcal{P}_{f}(\Omega) / G} Z\left(G_{A}^{A}\right),
$$

where $\mathcal{P}_{f}(\Omega) / G$ denotes a set of orbit representatives for $G$ acting on the finite subsets of $\Omega ; G_{A}^{A}$ denotes the (finite) permutation group induced on $A$ by its setwise stabiliser in $G$; and $Z(H)$ is the ordinary cycle index of the finite permutation group $H$, defined by

$$
Z(H)=\frac{1}{|H|} \sum_{h \in H} s_{1}^{c_{1}(h)} s_{2}^{c_{2}(h)} \cdots,
$$

where $c_{i}(h)$ is the number of $i$-cycles of $h$ and $s_{1}, s_{2}, \ldots$ are indeterminates.

If $G$ is finite, we obtain nothing new:

$$
\tilde{Z}(G)=Z\left(G ; s_{i} \leftarrow s_{i}+1\right) .
$$

But the modified cycle index is defined for any oligomorphic permutation group, although there is no hope of defining the ordinary cycle index of an infinite permutation group.

\section{Theorem 4.1}

$$
\begin{aligned}
F_{G}(x) & =\tilde{Z}\left(G ; s_{i} \leftarrow x^{i}\right) \\
f_{G}(x) & =\tilde{Z}\left(G ; s_{1} \leftarrow x, s_{i} \leftarrow 1 \text { for } i>0\right)
\end{aligned}
$$

\subsection{General properties}

It is easy to see that passing from an oligomorphic group $G$ to its closure does not change the modified cycle index of $G$, and hence does not change the sequences $F_{n}$ and $f_{n}$ associated with $G$.

Furthermore, if $G$ is an oligomorphic permutation group on $\Omega$, then there exists an oligomorphic permutation group $G^{\prime}$ on a countable set $\Omega^{\prime}$ which has the same modified cycle index (and hence the same numbers $F_{n}$ and $f_{n}$ ) as $G$. This follows from the downward Löwenheim-Skolem theorem of model theory. For in a suitable countable first-order language we can state that $G$ is a group, that $G$ acts on $\Omega$, and that $G$ has $f_{n}$ orbits on $n$-subsets of $\Omega$ with the appropriate group induced on a set in the $i$ th orbit, for all $n$. This theory has a model, by assumption, and so has a countable model. (Indeed, we may assume that $G^{\prime}$ is countable as well; but if we replace $G^{\prime}$ by its closure, this is no longer true.)

A first-order theory is $\aleph_{0}$-categorical if it has a unique countable model (up to isomorphism). It turns out that the closed oligomorphic permutation groups of countable degree are precisely the automorphism groups of countable models of $\aleph_{0}$-categorical theories; this is the celebrated theorem of Engeler, Ryll-Nardzewski, and Svenonius. 
Theorem 4.2 Let $M$ be a countable first-order structure. Then $\operatorname{Th}(M)$ is $\aleph_{0}$ categorical if and only if $\operatorname{Aut}(M)$ is oligomorphic. If this happens, then every $n$-type over $\operatorname{Th}(M)$ is realised in $M$, and the set of realising $n$-tuples is an orbit of $\operatorname{Aut}(M)$ on $M^{n}$.

Here, an $n$-type is a set $S$ of $n$-variable formulae in the language of $\operatorname{Th}(M)$ which is maximal with respect to the property that

$$
\operatorname{Th}(M) \cup\left\{\left(\exists x_{1}, \ldots, x_{n}\right) \phi\left(x_{1}, \ldots, x_{n}\right): \phi \in S\right\}
$$

is consistent. It is realised in $M$ if there exists a realising $n$-tuple $\left(a_{1}, \ldots, a_{n}\right)$ (with $a_{i} \in M$ for all $\left.i\right)$ such that $\phi\left(a_{1}, \ldots, a_{n}\right)$ is true in $M$.

So the sequences $F_{n}^{*}(G)$ for oligomorphic permutation groups $G$ are precisely the sequences counting types in $\aleph_{0}$-categorical theories.

Example 4.3 1. According to Cantor's theorem, the ordered set $\mathbb{Q}$ of rational numbers is characterised as the unique countable dense total order without endpoints. All the conditions except countability are first-order. Thus $\mathbb{Q}$ as ordered set is $\aleph_{0}$-categorical, and $A=\operatorname{Aut}(\mathbb{Q},<)$ is oligomorphic. Indeed, it is easy to see that $A$ is transitive on $n$-element subsets of $\mathbb{Q}$ for all $n$, and has $n$ ! orbits on ordered $n$-tuples of distinct elements of $\mathbb{Q}$.

2. Condition $(*)$, which we met in the discussion of the random graph $R$, is a conjunction of first-order sentences (one for each pair of values of $|U|$ and $|V|$ ). Thus, $R$ is $\aleph_{0}$-categorical, and $\operatorname{Aut}(R)$ is oligomorphic. Indeed, $F_{n}(\operatorname{Aut}(R))$ is the number of labelled $n$-vertex graphs (namely $2^{n(n-1) / 2}$ ), and $f_{n}(\operatorname{Aut}(R))$ is the number of unlabelled $n$-vertex graphs (that is, graphs up to isomorphism).

In general, let $\mathcal{C}$ be an isomorphism-closed class of finite structures. When we refer to unlabelled structures in $\mathcal{C}$, we mean isomorphism classes of $\mathcal{C}$-structures; when we refer to labelled structures in $\mathcal{C}$, we mean structures with underlying set $\{1, \ldots, n\}$. (For example, there are four unlabelled graphs and eight labelled graphs on 3 vertices). Now, if $\mathcal{C}$ is the age of a countable homogeneous relational structure $M$, then the numbers of unlabelled and labelled $n$-element structures in $\mathcal{C}$ are $f_{n}(\operatorname{Aut}(M))$ and $F_{n}(\operatorname{Aut}(M))$ respectively. So our orbit-counting problems generalise standard combinatorial enumeration problems for Fraïssé classes.

In this connection, I remark that an age is a species in the sense of Joyal [36]. If $\mathcal{C}=\operatorname{Age}(M)$ where $M$ is homogeneous, then the modified cycle index of $\operatorname{Aut}(M)$ is the cycle index of the species $\mathcal{C}$, in Joyal's terminology.

\subsection{Highly set-transitive groups}

Fraïssé classes give rise to a wide class of combinatorial enumeration problems, and raise the possibility of general results about the behaviour of the counting functions for such classes, which we now discuss. First, note that, unlike other counting problems in group theory (such as word growth or subgroup growth of finitely generated groups), there is no upper bound to the growth rate of $\left(f_{n}(G)\right)$ or $\left(F_{n}(G)\right)$. For given any sequence $\left(a_{n}\right)$, however rapidly growing, consider a relational language 
with $a_{n}$ relations of arity $n$, for all $n$, and let $\mathcal{C}$ be the class of finite structures in which each $n$-ary relation is an $n$-uniform hypergraph. (This condition ensures that there are only finitely many $n$-element structures in $\mathcal{C}$, up to isomorphism.) Then $\mathcal{C}$ is a Fraïssé class, and its Fraïssé limit $M$ satisfies $f_{n}(\operatorname{Aut}(M)) \geq 2^{a_{n}}$ for all $n$.

Indeed, it is the slowest growth that is the most interesting!

Of course, the slowest possible growth is no growth at all. Obviously $F_{n}(G)=1$ for all $n$ if and only if $G$ is highly transitive; so the only closed group with this property is the symmetric group (though there are many other highly transitive groups, some of which we have seen). For $f_{n}(G)$, things are more interesting. We say that a permutation group $G$ on $\Omega$ is highly set-transitive if $f_{n}(G)=1$ for all $n$. Now the following holds [11]:

Theorem 4.4 Let $G$ be a closed highly homogeneous permutation group of countable degree. Then $G$ is one of the following five groups:

$A$ : the group of order-preserving permutations of $\mathbb{Q}$;

$B$ : the group of order-preserving or order-reversing permutations of $\mathbb{Q}$;

$C$ : the group of permutations preserving the circular order on the set of complex roots of unity;

$D$ : the group of permutations preserving or reversing the circular order on the set of complex roots of unity;

$S:$ the symmetric group.

This theorem has wider implications. It follows that any highly homogeneous group of countable degree is contained as a dense subgroup in one of these five groups. Moreover, using the downward Löwenheim-Skolem theorem, it shows that a highly homogeneous but not highly transitive group of arbitrary infinite degree preserves or reverses a linear or circular order. Also, the theorem shows that the only reducts of $A$ are the groups $A, B, C, D, S$.

Throughout the remainder of this article, the symbols $A, B, C, D, S$ will be used in the sense of this theorem.

The modified cycle index for each of these groups can be calculated easily, since there is just one orbit on $n$-sets for each $n$, and the group induced on an $n$-set by its setwise stabiliser is respectively the trivial group, the cyclic group of order 2 with at most one fixed point (if $n>1$, the regular cyclic group of order $n$, and the dihedral group of order $2 n$ (if $n>2$ ). In particular, we have the following:

\begin{tabular}{ccc}
$G$ & $F_{G}(x)$ & $f_{G}(x)$ \\
\hline$A$ & $1 /(1-x)$ & $1 /(1-x)$ \\
$C$ & $1-\log (1-x)$ & $1 /(1-x)$ \\
$S$ & $\mathrm{e}^{x}$ & $1 /(1-x)$
\end{tabular}


Note the similarity between the conclusions of Theorem 4.4 and Thomas' Theorem 2.12 on the reducts of $\operatorname{Aut}(R)$. The analogy goes deeper, since the circular order can be produced by a switching-like construction, as John MacDermott pointed out to me. (It was this insight which sparked my interest in infinite permutation groups in the 1970s.)

\subsection{Direct and wreath products}

Next I turn to some constructions of new oligomorphic groups from old.

Let $G_{1}$ and $G_{2}$ be permutation groups on $\Omega_{1}$ and $\Omega_{2}$ respectively. The direct product $G_{1} \times G_{2}$ has two natural actions:

- the intransitive action on the disjoint union of $\Omega_{1}$ and $\Omega_{2}$, where

$$
\alpha\left(g_{1}, g_{2}\right)=\alpha g_{i} \quad \text { if } \alpha \in \Omega_{i}, \quad i=1,2 .
$$

- the product action on the Cartesian product of $\Omega_{1}$ and $\Omega_{2}$, where

$$
\left(\alpha_{1}, \alpha_{2}\right)\left(g_{1}, g_{2}\right)=\left(\alpha_{1} g_{1}, \alpha_{2} g_{2}\right) .
$$

If $G_{1}$ and $G_{2}$ are oligomorphic, then $G_{1} \times G_{2}$ is oligomorphic in each of these actions. For the intransitive action, things are simple; the modified cycle index is multiplicative:

$$
\tilde{Z}\left(G_{1} \times G_{2}\right)=\tilde{Z}\left(G_{1}\right) \tilde{Z}\left(G_{2}\right) .
$$

From this, of course, it follows that the generating functions $F(x)$ and $f(x)$ for orbits on ordered tuples or unordered sets are also multiplicative.

The product action is more complicated. Here, the number of orbits on arbitrary $n$-tuples is multiplicative:

$$
F_{n}^{*}\left(G_{1} \times G_{2}\right)=F_{n}^{*}\left(G_{1}\right) F_{n}^{*}\left(G_{2}\right) .
$$

This relation does not easily translate into a relation for the generating functions. The composition of modified cycle index works as follows [19]: define a 'multiplication' $\circ$ of indeterminates by

$$
s_{i} \circ s_{j}=\left(s_{\operatorname{lcm}(i, j)}\right)^{\operatorname{gcd}(i, j)},
$$

and extend multiplicatively to arbitrary monomials and then additively to arbitrary polynomials. Then

$$
Z(G \times H)=Z(G) \circ Z(H) .
$$

It is possible to deduce the multiplicativity of $F_{n}^{*}$ from this.

Example 4.5 1. For the group $G=S \times S$ in the product action, the asymptotic behaviour of the sequence $\left(f_{n}(G)\right)$ is not known.

2. Consider the group $G=A \times A$ in the product action. Then $f_{n}(G)$ is equal to the number of zero-one matrices with $n$ ones, having no row or column consisting entirely of zeros. The asymptotics of this sequence have been worked out [22]: we have

$$
f_{n}(G) \sim \frac{n !}{4} e^{-\frac{1}{2}(\log 2)^{2}} \frac{1}{(\log 2)^{2 n+2}} .
$$


Now we turn to wreath products. The definition of the (unrestricted) wreath product $G_{1}$ Wr $G_{2}$ depends on the action of $G_{2}$ on $\Omega_{2}$ : the base group consists of the Cartesian product of $\Omega_{2}$ copies of $G_{1}$, and the top group $G_{2}$ acts on the base group by permuting the indices of the factors. Thus, elements of the base group are functions $f: \Omega_{2} \rightarrow G_{1}$, and

$$
f^{g_{2}}\left(\alpha_{2}\right)=f\left(\alpha_{2} g_{2}^{-1}\right) .
$$

There are two natural actions of the wreath product:

- the imprimitive action on $\Omega_{1} \times \Omega_{2}$ : the base group acts by

$$
\left(\alpha_{1}, \alpha_{2}\right) f=\left(\alpha_{1} f\left(\alpha_{2}\right), \alpha_{2}\right)
$$

and the top group acts on the second coordinate.

- the power action (also called the product action) on the set $\Omega_{1}^{\Omega_{2}}$ of functions $\phi: \Omega_{2} \rightarrow \Omega_{2}$, where the base group acts by

$$
(\phi f)\left(\alpha_{2}\right)=\phi\left(\alpha_{2}\right) f\left(\alpha_{2}\right)
$$

and the top group acts by

$$
\left(\phi g_{2}\right)\left(\alpha_{2}\right)=\phi\left(\alpha_{2} g_{2}^{-1}\right)
$$

If $G_{1}$ and $G_{2}$ are oligomorphic, then $G_{1} \mathrm{Wr} G_{2}$ in the imprimitive action is oligomorphic. Its modified cycle index is obtained from that of $G_{2}$ by substituting $\tilde{Z}\left(s_{i}, s_{2 i}, \ldots\right)-1$ for $s_{i}$, for $i=1,2, \ldots$. It follows that

$$
F_{G_{1} \mathrm{Wr} G_{2}}(x)=F_{G_{2}}\left(F_{G_{1}}(x)-1\right) ;
$$

however, $f_{G_{1} \mathrm{Wr} G_{2}}$ is not expressible in terms of $f_{G_{1}}$ and $f_{G_{2}}$ alone. (It can be obtained from the modified cycle index of $G_{2}$ by substituting $f_{G_{1}}\left(x^{i}\right)-1$ for $s_{i}$ for $i=1,2, \ldots$.)

Exercise: Show that the groups $C \mathrm{Wr} S$ and $A$ have the same numbers of orbits on ordered $n$-tuples of distinct elements for all $n$. (Combinatorially, the orbits of these two groups correspond to permutations and linear orders of $\{1, \ldots, n\}$ respectively; any permutation can be written as a disjoint union of cycles.)

The power action of $G_{1} \mathrm{Wr} G_{2}$ is oligomorphic if and only if $G_{1}$ is oligomorphic and $G_{2}$ is a finite permutation group acting on a finite set. (If $\Omega_{2}$ is finite, then $G_{1} \mathrm{Wr} G_{2}$ contains the direct product of $\left|\Omega_{2}\right|$ copies of $G_{1}$ in the product action, and hence is oligomorphic. But if $\Omega_{2}$ is infinite, then pairs of functions $\left(\phi, \phi^{\prime}\right)$ agreeing in $i$ positions lie in different orbits for $i=1,2, \ldots$ )

The problem of expressing the modified cycle index of $G_{1} \mathrm{Wr} G_{2}$ (in the power action) in terms of those of the factors has not been solved. 


\subsection{Growth rates}

One of the most basic facts about the sequences $\left(f_{n}\right)$ and $\left(F_{n}\right)$ is that they are non-decreasing. In the case of $\left(F_{n}\right)$, this is clear: there is a map from $(n+1)$-tuples to $n$-tuples (taking the initial segment) which commutes with the action of the group, and is onto. We see that $F_{n}=F_{n+1}$ if and only if $G$ is $(n+1)$-transitive.

The inequality $f_{n} \leq f_{n+1}$ is more subtle: two proofs are known, one using linear algebra, the other Ramsey's theorem. The characterisation of the case of equality here is not known. It is known that a primitive group with $f_{n}=f_{n+1}$ must be 2-homogeneous, and that $f_{n}$ is bounded by a function of $n$; it is conjectured that there is an absolute bound on $n$ [23]. (We will soon see an example of a primitive group with $f_{6}=f_{7}=2$.)

For the symmetric group $S$, we have $f_{n}(S)=F_{n}(S)=1$ for all $n$. Thus, $f_{S}(x)=(1-x)^{-1}$. So if $S^{k}$ denotes the direct product of $k$ copies of $S$ with the intransitive action, then $f_{S^{k}}(x)=(1-x)^{-k}$, from which we see by the negative binomial theorem that

$$
f_{n}\left(S^{k}\right)=\left(\begin{array}{c}
n+k-1 \\
k-1
\end{array}\right)
$$

a polynomial in $n$ with degree $k-1$. Examples of transitive but imprimitive groups with polynomial growth of $\left(f_{n}\right)$ can also easily be constructed. However, for the group $G=S$ Wr $S$ (with the imprimitive action), we see that $f_{n}(G)=p(n)$, the number of partitions of $n$; for this imprimitive group, we have fractional exponential growth: precisely,

$$
p(n) \sim \frac{1}{4 n \sqrt{3}} \mathrm{e}^{\pi \sqrt{2 n / 3}} .
$$

What happens for primitive groups? The first result asserts the existence of a wide gap, between constant and exponential growth.

Theorem 4.6 There is an absolute constant $c>1$ such that, if $G$ is primitive but not highly homogeneous, then

(a) the numbers of orbits on $n$-sets satisfy $f_{n} \geq c^{n} / p(n)$ for some polynomial $p$;

(b) the numbers of orbits on $n$-tuples of distinct elements satisfy $F_{n} \geq c^{n} n ! / p(n)$ for some polynomial $p$.

The first part of the theorem is due to Macpherson [40], who gave the value $c=\sqrt[5]{2}=1.148 \ldots$. The second part is due to Merola [46], who also improved Macpherson's constant to $c=1.324 \ldots$... No counterexamples are known to the conjecture that both parts hold with $c=2$.

The growth rates of the sequences $\left(f_{n}\right)$ and $\left(F_{n}\right)$ appear to have a great deal of structure; little is known, but examples suggest some conjectures. One of the most challenging conjectures is to prove that various 'obvious' limits, such as $\lim \left(\log f_{n}\right) /(\log n)$ (for polynomial growth), $\lim \left(\log \log f_{n}\right) /(\log n)$ (for fractional exponential growth), or $\lim \left(\log f_{n}\right) / n$ (for exponential growth), actually exist. For primitive groups with exponential growth, as noted above, we have no examples where $\lim \left(f_{n}\right)^{1 / n}$ is strictly less than 2 (assuming that it is not 1); and indeed, only countably many values for this limit are known. 
Example 4.7 Here are two examples of primitive groups where $f_{n}$ grows exponentially.

1. A tournament is a directed graph in which each pair of distinct vertices is joined by a directed edge in just one direction. A tournament is said to be a local order if it does not contain a 4-point subtournament consisting of a vertex dominating or dominated by a 3 -cycle. The finite local orders form a Fraïssé class, whose Fraïssé limit $T$ is more easily described as follows. Take a countable dense set of points on the unit circle containing no antipodal pair of points. (If we consider the set of all complex roots of unity, and randomly choose one out of each pair $\{\omega,-\omega\}$, then the resulting set is dense with probability 1.) Now put a directed edge from $x$ to $y$ if the shorter arc from $x$ to $y$ is in the anticlockwise direction. The result is the universal homogeneous local order $T$. We see that $\operatorname{Aut}(T)$ is 2 -set transitive, hence primitive; and $f_{n}(\operatorname{Aut}(T))$ is equal to the number of isomorphism types of $n$-vertex local order, which is asymptotically $2^{n-1} / n$. (In fact, by taking the larger group $G$ of permutations which preserve or reverse the edge directions, we obtain $f_{n}(G) \sim 2^{n-2} / n$, the slowest known growth rate for a primitive but not highly set-transitive group.

2. A boron tree is a finite tree in which every vertex has valency 1 or 3. (Boron trees describe the analogue of hydrocarbons in a boron-based chemistry.) On the set of leaves of a boron tree, we can define a 4-place relation as follows. Given four points $a, b, c, d$, there is a unique partition into two sets of size 2 such that the paths joining vertices in the same set do not intersect. Write $R(a, b ; c, d)$ if this partition is $a b \mid c d$. (See Figure 1 ). The relational structures obtained in this way form a Fraïssé class; the boron tree is uniquely recoverable from the quaternary relation on the set of leaves. Thus, if $G$ is the automorphism group of the Fraïssé limit, then $f_{n}(G)$ is equal to the number of boron trees with $2 n-2$ vertices, which is asymptotically $A n^{-5 / 2} c^{n}$, where $c=2.483 \ldots$. Note in passing that this group is 3 -transitive but not 4 transitive, is 5 -set transitive, and has $f_{6}=f_{7}=2$. (All these assertions can be proved by drawing diagrams like Figure 1.)

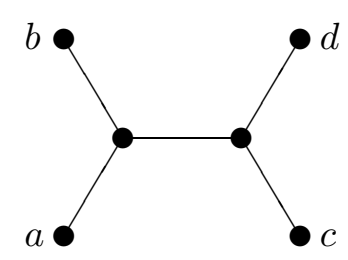

Figure 1. A boron tree

It has been observed that most of the primitive oligomorphic groups in which $\left(f_{n}\right)$ exhibits exponential growth (including the above examples) are associated either with linear or circular orders or with trees (or some combination of these). Recently, a conceptual framework has been found which includes all these examples [33]: they are automorphism groups of $\aleph_{0}$-categorical, weakly o-minimal structures (those 
which are linearly ordered so that every definable set is a finite union of convex subsets). However, we are far from having a result asserting that any group with exponential growth of $\left(f_{n}\right)$ is of this form!

Known primitive groups at first appear to to show that there is a gap between exponential and factorial growth, and that there is a spectrum of possible values of $\lim \left(f_{n} / n !\right)^{1 / n}$ (that is, values of $c$ for which the growth is roughly $\left.c^{n} n !\right)$. Some results have been established under model-theoretic hypotheses related to stability, such as the strict order property, by Macpherson [41]. However, the group $G=$ $S \mathrm{Wr} S_{2}$, with the power action, has the property that $f_{n}(G)$ grows faster than exponentially but slower than factorially. The precise asymptotic behaviour is not known. A similar remark implies to the symmetric group in its action on 2-sets. (For this group $G, f_{n}(G)$ is the number of graphs with $n$ edges and no isolated vertices, up to isomorphism.)

Problem 4.8 What is the asymptotic behaviour of $f_{n}(G)$ for the two groups $G$ in the preceding paragraph?

\subsection{Graded algebras}

The numbers $f_{n}$ have another interpretation, as the coefficients in the Hilbert series of a graded algebra.

Given a set $\Omega$, the reduced incidence algebra of subsets of $\Omega$ is defined as follows. The $n$th homogeneous component $V_{n}$ is the space of functions from $\left(\begin{array}{l}\Omega \\ n\end{array}\right)$ to $\mathbb{C}$, with pointwise addition. Multiplication is the map $V_{n} \times V_{m} \rightarrow V_{n+m}$ defined as follows: for $f_{1} \in V_{n}, f_{2} \in V_{m}$, and $X \in\left(\begin{array}{c}\Omega \\ n+m\end{array}\right)$, we have

$$
\left(f_{1} f_{2}\right)(X)=\sum_{Y \in\left(\begin{array}{l}
X \\
n
\end{array}\right)} f_{1}(Y) f_{2}(X \backslash Y) .
$$

It is easily verified that

$$
A=\bigoplus_{n \geq 0} V_{n}
$$

is a commutative associative $\mathbb{N}$-graded algebra.

Now if $G$ is a permutation group on $\Omega$, then there is a natural action of $G$ on $V_{n}$, and hence on $A$; we let

$$
A^{G}=\bigoplus_{n \geq 0} V_{n}^{G}
$$

be the algebra of $A$-fixed points. Clearly $\operatorname{dim}\left(V_{n}^{G}\right)=f_{n}$ if this number is finite.

The structure of $A$ is known in some cases:

1. If $G=S$ (or indeed if $G$ is highly homogeneous), then $A^{G}$ is the polynomial algebra in a single generator $e$, the constant function with value 1 in $V_{1}$.

2. If $G=G_{1} \times G_{2}$ in its intransitive action, then $A^{G}=A^{G_{1}} \otimes_{\mathbb{C}} A^{G_{2}}$. Hence, for $G=S^{n}$ in the intransitive action, $A^{G}$ is a polynomial ring in $n$ generators of degree 1 . 
3. If $G=S \mathrm{Wr} H$ in its imprimitive action, where $H$ is a finite permutation group, then $A^{G}$ is isomorphic to the algebra of invariants of the linear group $H$. In particular, if $G=S \mathrm{Wr} S_{n}$, then $A^{G}$ is a polynomial ring in generators of degree $1,2, \ldots, n$.

4. Suppose that $G=\operatorname{Aut}(M)$, where $M$ is the Fraïssé limit of a class $\mathcal{C}$ of finite structures. If there are notions of 'connectedness' and 'involvement' in $\mathcal{C}$ satisfying a few simple axioms, then $A^{G}$ is a polynomial algebra generated by the connected structures in $\mathcal{C}$. (For details, see [15].) Here are three special cases.

- If $\mathcal{C}$ consists of all finite graphs, so that $M$ is the random graph, then the polynomial generators of $A^{G}$ correspond to the connected graphs.

- Suppose that elements of $\mathcal{C}$ are totally ordered sets coloured with $m$ colours. Any such structure is coded by a word in an $m$-letter alphabet. Then $A^{G}$ is the shuffle algebra [55, p. 24], and the polynomial generators are the Lyndon words. (The fact that the shuffle algebra is polynomial was proved by Radford [53].)

- Let $G=H \mathrm{Wr} S$, in its imprimitive action. Then $A^{G}$ is a polynomial algebra with $f_{n}(H)$ generators of degree $n$ for each $n$; the connected structures are those contained within a block of imprimitivity.

In general, little is known about the structure of $A^{G}$. An old conjecture of mine asserts that, if $G$ has no finite orbits on $\Omega$, then $A^{G}$ is an integral domain. (This conclusion would have some implications for the growth of the sequence $\left(f_{n}(G)\right)$.) It is known that the property that $A^{G}$ is an integral domain is preserved by passing to overgroups or transitive extensions [16].

If $A^{G}$ is a polynomial algebra with $a_{n}$ homogeneous generators of degree $n$ for each $n$, then the sequences $\left(a_{n}\right)$ and $\left(f_{n}\right)$ determine each other:

$$
\sum_{n \geq 0} f_{n} x^{n}=\prod_{k \geq 1}\left(1-x^{k}\right)^{-a_{k}}
$$

There are some permutation groups $G$ for which the structure of $A^{G}$ is not known, but the numbers $a_{n}$ computed from this formula on the assumption that it is a polynomial algebra have a combinatorial interpretation. Any such group provides an interesting puzzle, whether or not $A^{G}$ turns out to be a polynomial algebra: if it is, we have to match the generators with the combinatorial objects counted by $\left(a_{n}\right)$; if not, we have to explain the apparent coincidence.

For example, let $G$ be the group of switching automorphisms of the random graph $R$ (see Theorem 2.12). It is known that $f_{n}(G)$ is equal to the number of even graphs (graphs with all vertex degrees even) on $n$ vertices $[45,12]$; so $a_{n}$ is equal to the number of Eulerian graphs (connected even graphs) on $n$ vertices. Now $A^{G} \subseteq A^{\mathrm{Aut}(R)}$, and $A^{\mathrm{Aut}(R)}$ is a polynomial ring, so $A^{G}$ is an integral domain; but it is not known whether it is itself a polynomial ring.

Problem 4.9 For the above group $G$, is $A^{G}$ a polynomial ring? 


\section{The Urysohn space}

We have seen that the techniques used by Erdös and Rényi to prove their remarkable theorem about the random graph had been developed by Fraïssé more than ten years earlier for arbitrary relational structures. In fact, Fraïssé was not the first to do this.

In a posthumously-published paper, P. S. Urysohn [67] constructed a remarkable metric space:

Theorem 5.1 There exists a metric space $\mathbb{U}$, unique up to isometry, with the following properties:

- $\mathbb{U}$ is a Polish space (a complete separable metric space);

- $\mathbb{U}$ is homogeneous (every isometry between finite subspaces extends to an isometry of $\mathbb{U})$;

- $\mathbb{U}$ is universal (every Polish space can be embedded isometrically into $\mathbb{U}$ ).

It is interesting to compare this theorem with Fraïssé's. The theorem of Fraïssé deals only with countable objects. A universal Polish space clearly cannot be countable, since it must embed the real numbers. The replacement for countability is the condition of separability. (A metric space is separable if it has a countable dense subset).

With hindsight, can we construct $\mathbb{U}$ by Fraïssé's method? Clearly we can't do so directly, because it is uncountable. (Indeed, there are uncountably many twoelement metric spaces.) However, we can proceed as follows. Let $\mathcal{C}$ be the class of rational metric spaces, those for which all distances are rational. It is relatively easy to show that $\mathcal{C}$ is a Fraïssé class. We denote its Fraïssé limit by $Q \mathbb{U}$, and call it the rational Urysohn space. Then $\mathbb{U}$ is the completion of $Q \mathbb{U}$.

Vershik [69] showed the analogue of the Erdős-Rényi theorem, namely, that $\mathbb{U}$ is the random Polish space. Of course, the interpretation of this statement requires some care. Suppose that we build a countable metric space by adding one point at a time. At the $n$th stage, we have points $a_{0}, \ldots, a_{n-1}$, and wish to add a point $a_{n}$ with specified distances from the existing points. The vector $\left(x_{0}, \ldots, x_{n-1}\right)$, where $x_{i}=d\left(a_{n}, a_{i}\right)$, must satisfy the conditions

$$
\left|x_{i}-x_{j}\right| \leq d\left(a_{i}, a_{j}\right) \leq x_{i}+x_{j}
$$

as well as $x_{i} \geq 0$ for all $i$. (We allow non-strict inequality, but this will be very unlikely). The set of such admissible vectors then lies in an unbounded region of the positive orthant of $\mathbb{R}^{n}$ defined by these inequalities. Vershik showed that, if the vector of distances is chosen randomly from this set at each stage according to any of a wide range of natural probability distributions, then the completion of the resulting countable space is isometric to $\mathbb{U}$ with probability 1 .

In the paper [24], the authors attempted to apply some of the methods used to find subgroups of $\operatorname{Aut}(R)$ to the isometry group of $\mathbb{U}$. I now describe some of the results of this paper and some open problems. Let $\operatorname{Isom}(M)$ denote the isometry group of the metric space $M$. We note that $\operatorname{Isom}(Q \mathbb{U})$ is a dense subgroup of $\operatorname{Isom}(\mathbb{U})$ (in the topology induced by the product topology on $\mathbb{U}^{\mathbb{U}}$ ). 
Bounded and unbounded isometries An isometry $g$ of a metric space $M$ is bounded if there is a number $c$ such that $d(x, x g) \leq c$ for all $x \in M$. The bounded isometries form a normal subgroup BIsom $(M)$ of the group of all isometries. In the case of the Urysohn space, we have

$$
1 \neq \operatorname{BIsom}(\mathbb{U}) \neq \operatorname{Isom}(\mathbb{U}),
$$

but $\operatorname{BIsom}(\mathbb{U})$ is dense in $\operatorname{Isom}(\mathbb{U})$.

Problem 5.2 Is it true that

(a) $\operatorname{BIsom}(\mathbb{U})$ and $\operatorname{Isom}(\mathbb{U}) / B \operatorname{Bsom}(\mathbb{U})$ are simple groups, and

(b) $\operatorname{Isom}(\mathbb{U})$ is simple as a topological group?

The Tits method The factor group $\operatorname{Isom}(Q \mathbb{U}) / \operatorname{BIsom}(Q \mathbb{U})$ contains a nonabelian free subgroup. Hence the method of Tits can be used to show that

Theorem 5.3 Isom(U) contains a dense free subgroup.

Cyclic automorphisms A method like that for $R$ (but significantly more complicated) shows:

Theorem 5.4 $Q \mathbb{U}$ has an isometry $\sigma$ which permutes all its points in a single cycle: indeed, there are uncountably many conjugacy classes of such isometries.

Such a $\sigma$ can be regarded as an isometry of $\mathbb{U}$, all of whose orbits are dense. (This suggests the possibility of finding interesting dynamical systems here.) Moreover, the closure of the group generated by such a $\sigma$ is an abelian group acting transitively on $\mathbb{U}$; so the space $\mathbb{U}$ has an abelian group structure (indeed, many such structures).

The closure of an infinite cyclic group is abelian, but is not necessarily torsionfree. It is not known what sort of torsion these groups may have.

Problem 5.5 Describe the possible structure of the closure of a cyclic group of isometries of $\mathbb{U}$ with dense orbits.

Other regular groups The results obtained here are much more restricted. The sum total of our knowledge is the following: The countable elementary abelian 2group acts regularly on $Q \mathbb{U}$, but the countable elementary abelian 3-group cannot act on $\mathbb{U}$ with a dense orbit.

The latter fact is rather easy to prove, as follows. Suppose that we have such an action of this group $A$. Since the stabiliser of a point in the dense orbit is trivial, we can identify the points of the orbit with elements of $A$ (which we write additively).

Choose $x \neq 0$ and let $d(0, x)=\alpha$. Then $\{0, x,-x\}$ is an equilateral triangle with side $\alpha$. Since $\mathbb{U}$ is universal and $A$ is dense, there is an element $y$ such that $d(x, y), d(-x, y) \approx \frac{1}{2} \alpha$ and $d(0, y) \approx \frac{3}{2} \alpha$. (The approximation is to within a given $\epsilon$ chosen smaller than $\frac{1}{6} \alpha$ ). Then the three points $0, y, x-y$ form a triangle with sides approximately $\frac{3}{2} \alpha, \frac{1}{2} \alpha, \frac{1}{2} \alpha$, contradicting the triangle inequality.

An open problem is to find other examples of groups which do (or don't) have a regular action on $Q \mathbb{U}$ (or another countable dense subset of $\mathbb{U}$ ). 
Very recently, Nešetřil [48] has shown that finite metric spaces form a Ramsey class. It follows from the results of Kechris et al. [37] that the isometry group of the "ordered" version of $\mathbb{U}$ is extremely amenable.

\section{$6 \quad$ Further topics}

\subsection{Finitary permutation groups}

A permutation group is finitary if its elements move only finitely many points. The finitary permutations of $\Omega$ form a group, the finitary symmetric group, which is locally finite and highly transitive, and is normal in the symmetric group on $\Omega$. Indeed, if $\Omega$ is countable, then the only normal subgroups of the symmetric group, apart from itself and the trivial group, are the finitary symmetric group and the alternating group (consisting of all the finitary even permutations).

Wielandt [72], extending a theorem of Jordan to the infinite, showed:

Theorem 6.1 A primitive infinite permutation group which contains a non-identity finitary permutation must contain the alternating group (and hence is highly transitive).

This result is usually referred to as the Jordan-Wielandt Theorem.

Extending further, Neumann $[49,50]$ developed a structure theory for finitary permutation groups. This has been further extended to finitary linear groups and even to finitary automorphism groups of modules over arbitrary rings.

\subsection{Jordan groups}

This topic for finite permutation groups dates from the nineteenth century, although definitive results had to await the Classification of Finite Simple Groups. The picture is much richer in the infinite case.

Let $G$ be a transitive permutation group on $\Omega$. A subset $\Delta$ of $\Omega$, containing more than one point, is a Jordan set if $G$ contains a subgroup $H$ which fixes the complement of $\Delta$ pointwise and acts transitively on $\Omega$. Of course, if $G$ is $(n+1)$ transitive, then any set whose complement contains just $n$ points is a Jordan set; such a Jordan set is called improper. A Jordan group is a transitive permutation group having at least one proper Jordan set. Usually we consider only primitive Jordan groups.

For example, the Jordan-Wielandt Theorem, discussed in the last subsection, shows that a primitive group with a finite Jordan set must contain the alternating group. Dually, Neumann [51] showed that a primitive Jordan group with a cofinite proper Jordan set is a projective or affine group over a finite field or is highly transitive. (This paper is recommended for its treatment of finite Jordan groups.)

These examples, however, give no hint of the richness of general Jordan groups. For example, the group of homeomorphisms of a manifold (of dimension at least 2) is a Jordan group: any open ball is a Jordan set, since we can permute its points transitively while fixing its boundary. This group is highly transitive. To avoid 
such examples, we make the further restriction that our Jordan group is not highly transitive.

Example 6.2 1 . The group $A$ of order-preserving permutations of the rational numbers is a Jordan group: the stabiliser of the point $\alpha$ is isomorphic to $A \times A$ (where the factors act transitively on the Jordan sets $\{x: x<\alpha\}$ and $\{x ; x>\alpha\}$.

2. The group associated with boron trees in the last section is a Jordan group. We can think of the points as being the ends of a dense treelike object; given an internal vertex, the ends fall into three classes each admitting a transitive group fixing the others pointwise.

3. As in the finite case, a projective or affine group (of finite or infinite dimension over a finite or infinite field) is a Jordan group, the Jordan sets being complements of subspaces.

A remarkable classification of the infinite Jordan groups which are primitive but not highly transitive was achieved by Adeleke and Macpherson [1]. They show that such a group preserves either a relational structure of a special types or a "limit" of such structures. The types are linear, circular and semilinear orders, general betweenness and separation relations, C- and D-relations, and Steiner systems. (See the memoir of Adeleke and Neumann [2] for a detailed description and axiomatisation of the types of relations which can appear.)

Note that there is a big overlap between these groups and two classes we have mentioned before: the primitive oligomorphic groups $G$ for which $\left(f_{n}(G)\right)$ grows exponentially; and the automorphism groups of the $\aleph_{0}$-categorical, weakly o-minimal structures. It is not clear why these overlaps occur.

\subsection{Maximal subgroups of $\operatorname{Sym}(\Omega)$}

In this section, unlike most of the rest of the paper, we do not assume that $\Omega$ is countable.

The O'Nan-Scott Theorem is usually stated as a result about finite primitive (or quasiprimitive) permutation groups. Yet in its original form [58], it was a classification of maximal subgroups of finite symmetric (or alternating) groups into several types: intransitive (subset stabilisers); imprimitive (partition stabilisers); affine; wreath products with product action; diagonal groups; and almost simple groups. Now we know much more about which groups of each type are actually maximal.

For infinite symmetric groups, things are more complicated: a classification based on the socle is not possible since there may be no minimal normal subgroup; and indeed, there may be subgroups of the symmetric group which lie in no maximal subgroup.

The first difficulty arises from the fact that $\operatorname{Sym}(\Omega)$ has non-trivial normal subgroups. If $H$ is a maximal subgroup of $\operatorname{Sym}(\Omega)$ and $\aleph_{0} \leq c \leq|\Omega|$, then either $\operatorname{BSym}_{c}(\Omega) \leq H$ or $\operatorname{BSym}_{c}(\Omega) H=\operatorname{Sym}(\Omega)$. In the first case, $H$ is highly transitive; in the second, Macpherson and Neumann [43] showed that there is a subset 
$\Delta$ with $|\Delta|<c$ whose setwise stabiliser in $\Omega$ induces the symmetric group on its complement.

It is clear that the setwise stabiliser of a finite set is a maximal subgroup of $\operatorname{Sym}(\Omega)$. In 1967, Richman [56] demonstrated two further classes of maximal subgroups: the setwise stabiliser of a finite set of equivalent ultrafilters; and the almost stabiliser of a partition into a finite number of sets of equal cardinality. (A filter is a family of sets closed under intersection and closed upwards but not containing the empty set; an ultrafilter is a filter which is maximal with respect to inclusion. An ultrafilter is principal if it consists of all sets containing a particular point of $\Omega$. Two ultrafilters are equivalent if one can be mapped to the other by a permutation of $\Omega$. Thus Richman's first class of groups includes the setwise stabilisers of finite sets. A partition $\pi$ is almost stabilised by $g$ if, for all $A \in \pi$, there exists $B \in \pi$ such that the symmetric difference of $A g$ and $B$ is finite.)

These two classes of maximal subgroups correspond roughly to the maximal intransitive and imprimitive subgroups of finite symmetric groups. Subsequently, several other classes of maximal subgroups of symmetric groups, corresponding in a similar rough sense to other O'Nan-Scott types, have been established [9, 26].

Peter Neumann raised the question: is every proper subgroup $H$ of $\operatorname{Sym}(\Omega)$ contained in a maximal subgroup? Macpherson and Praeger showed that this is the case if $H$ is not highly transitive. However, Baumgartner et al. [4] showed that it is consistent with the ZFC axioms for set theory that there exists a subgroup $T$ of $\operatorname{Sym}(\Omega)$, where $|\Omega|=\kappa$, for which the subgroups between $T$ and $\operatorname{Sym}(\Omega)$ form a well-ordered chain of order type $\kappa^{+}$. (This result is proved assuming GCH. Of course, all the intermediate groups are highly transitive. For independence results, see [59]. Note also that Macpherson and Neumann proved that a chain of proper subgroups with union $\operatorname{Sym}(\Omega)$ must have length greater than $|\Omega|$, so in a sense the result of Baumgartner et al. is best possible.)

If $H$ is a maximal subgroup of $\operatorname{Sym}(\Omega)$, then $\langle H, g\rangle=\operatorname{Sym}(\Omega)$ for all $g \notin H$. Galvin [30] proved the remarkable result that if $H$ is any subgroup such that $\langle H, B\rangle=\operatorname{Sym}(\Omega)$ for some set $B$ with $|B| \leq|\Omega|$, then there is some element $g$ such that $\langle H, g\rangle=\operatorname{Sym}(\Omega)$. Moreover, the order of $g$ can be chosen to be any preassigned even number greater than 2. (We saw earlier, while discussing the the Bergman-Shelah Theorem 2.10, that the hypothesis of Galvin's theorem hold if $\Omega$ is countable, $H$ is closed in $\operatorname{Sym}(\Omega)$, and the stabiliser of a finite tuple of points in $H$ has at least one infinite orbit on the remaining points.)

\section{References}

[1] S. A. Adeleke and H. D. Macpherson, Classification of infinite primitive Jordan groups, Proc. London Math. Soc. (3) 72 (1996), 63-123.

[2] S. A. Adeleke and P. M. Neumann, Relations related to betweenness: their structure and automorphisms, Memoirs Amer. Math. Soc. 131, no. 623, American Mathematical Society, Providence, RI, 1988.

[3] S. A. Adeleke and P. M. Neumann, Primitive permutation groups with primitive Jordan sets, J. London Math. Soc. (2) 53 (1996), 209-229.

[4] J. E. Baumgartner, S. Shelah and S. Thomas, Maximal subgroups of infinite symmetric groups, Notre Dame J. Formal Logic 34 (1993), 1-11. 
[5] G. M. Bergman and S. Shelah, Closed subgroups of the infinite symmetric group, preprint.

[6] M. Bhattacharjee and H. D. Macpherson, Strange permutation representations of free groups, J. Austral. Math. Soc. 74 (2003), 267-285.

[7] M. Bhattacharjee and H. D. Macpherson, A locally finite dense group acting on the random graph, Forum Math. 17 (2005), 513-517.

[8] A. Bonato, D. Delić and I. Dolinka, All countable monoids embed into the monoid of the infinite random graph, to appear.

[9] M. Brazil, J. Covington, T. Penttila, C. E. Praeger and A. R. Woods, Maximal subgroups of infinite symmetric groups, Proc. London Math. Soc. (3) 68 (1994), 77-111.

[10] M. Brown, Weak $n$-homogeneity implies weak (n-1)-homogeneity, Proc. Amer. Math. Soc. 10 (1959), 644-647.

[11] P. J. Cameron, Transitivity of permutation groups on unordered sets, Math. Z. 48 (1976), 127-139.

[12] P. J. Cameron, Cohomological aspects of two-graphs, Math. Z. 157 (1977), 101-119.

[13] P. J. Cameron, Oligomorphic Permutation Groups, London Math. Soc Lecture Notes 152, Cambridge University Press, Cambridge, 1990.

[14] P. J. Cameron, The random graph, pp. 331-351 in The Mathematics of Paul Erdös, (J. Nešetřil and R. L. Graham, eds.), Springer, Berlin, 1996.

[15] P. J. Cameron, The algebra of an age, pp. 126-133 in Model Theory of Groups and Automorphism Groups (ed. David M. Evans), London Mathematical Society Lecture Notes 244, Cambridge University Press, Cambridge, 1997.

[16] P. J. Cameron, On an algebra related to orbit-counting, J. Group Theory 1 (1998), 173-179.

[17] P. J. Cameron, Homogeneous Cayley objects, European J. Combinatorics 21 (2000), $745-760$.

[18] P. J. Cameron, The random graph has the strong small index property, Discrete Math. 291 (2005), 41-43.

[19] P. J. Cameron, D. A. Gewurz and F. Merola, Product action, preprint.

[20] P. J. Cameron and K. W. Johnson, An essay on countable B-groups, Math. Proc. Cambridge Philos. Soc. 102 (1987), 223-232.

[21] P. J. Cameron, P. M. Neumann and D. N. Teague, On the degrees of primitive permutation groups, Math. Z. 180 (1982), 141-149.

[22] P. J. Cameron, T. J. Prellberg, and D. S. Stark, Asymptotic enumeration of incidence matrices, preprint.

[23] P. J. Cameron and S. Thomas, Groups acting on unordered sets, Proc. London Math. Soc. (3) 59 (1989), 541-557.

[24] P. J. Cameron and A. M. Vershik, Some isometry groups of Urysohn space, preprint.

[25] G. Cherlin, The classification of countable homogeneous directed graphs and countable homogeneous $n$-tournaments, Memoirs Amer. Math. Soc. 621, American Mathematical Society, Providence, RI, 1998.

[26] J. Covington, H. D. Macpherson and A. D. Mekler, Some maximal subgroups of the symmetric groups, Quart. J. Math. Oxford (2) 47 (1996), 297-311.

[27] M. Droste, Structure of partially ordered sets with transitive automorphism groups, Memoirs Amer. Math. Soc. 57 (1985).

[28] P. Erdős and A. Rényi, Asymmetric graphs, Acta Math. Acad. Sci. Hungar. 14 (1963), 295-315.

[29] R. Fraïssé, Sur certains relations qui généralisent l'ordre des nombres rationnels, $C$. R. Acad. Sci. Paris 237 (1953), 540-542.

[30] F. Galvin, Generating countable sets of permutations, J. London Math. Soc. (2) 51 (1995), 232-242. 
[31] M. Hall Jr., On a theorem of Jordan, Pacific J. Math. 4 (1954), 219-226.

[32] C. W. Henson, A family of countable homogeneous graphs, Pacific J. Math. 38 (1971), 69-83.

[33] B. Herwig, H. D. Macpherson, G. Martin, A, Nurtazin and J. Truss, On $\aleph_{0}$-categorical weakly o-minimal structures, Ann. Pure Appl. Logic 101 (2000), 65-93.

[34] W. A. Hodges, I. M. Hodkinson, D. Lascar and S. Shelah, The small index property for $\omega$-stable, $\omega$-categorical structures and for the random graph, J. London Math. Soc. (2) 48 (1993), 204-218.

[35] E. Hrushovski, Extending partial isomorphisms of graphs, Combinatorica 12 (1992), 411-416.

[36] A. Joyal, Une theorie combinatoire des séries formelles, Advances Math. 42 (1981), $1-82$.

[37] A. S. Kechris, V. G. Pestov, and S. Todorcevic, Fraïssé limits, Ramsey theory, and topological dynamics of automorphism groups, Geometric and Functional Analysis 15 (2005), 106-189.

[38] A. H. Lachlan, Countable homogeneous tournaments, Trans. Amer. Math. Soc. 284 (1984), 431-461.

[39] A. H. Lachlan and R. E. Woodrow, Countable ultrahomogeneous undirected graphs, Trans. Amer. Math. Soc. 262 (1980), 51-94.

[40] H. D. Macpherson, Growth rates in infinite graphs and permutation groups, Proc. London Math. Soc. (3) 51 (1985), 285-294.

[41] H. D. Macpherson, Infinite permutation groups of rapid growth, J. London Math. Soc. (2) 35 (1987), 276-286.

[42] H. D. Macpherson, Sharply multiply homogeneous permutation groups, and rational scale types, Forum Math. 8 (1996), 501-507.

[43] H. D. Macpherson and P. M. Neumann, Subgroups of infinite symmetric groups, J. London Math. Soc. (2) 42 (1990), 64-84.

[44] H. D. Macpherson and C. E. Praeger, Subgroups of infinite symmetric groups, $J$. London Math. Soc (2) 42 (1990), 85-92.

[45] C. L. Mallows and N. J. A. Sloane, Two-graphs, switching classes, and Euler graphs are equal in number, SIAM J. Appl. Math. 28 (1975), 876-880.

[46] F. Merola, Orbits on $n$-tuples for infinite permutation groups, Europ. J. Combinatorics 22 (2001), 225-241.

[47] J. Nešetřil, Ramsey classes and homogeneous structures, Combinatorics, Probability and Computing 14 (2005), 171-189.

[48] J. Nešetřil, Metric spaces are Ramsey, to appear.

[49] P. M. Neumann, The lawlessness of finitary permutation groups, Arch. Math. 26 (1975), 561-566.

[50] P. M. Neumann, The structure of finitary permutation groups, Arch. Math. 27 (1976), $3-17$.

[51] P. M. Neumann, Some primitive permutation groups, Proc. London Math. Soc. (3) 50 (1985), 265-281.

[52] V. Pestov, Ramsey-Milman phenomenon, Urysohn metric spaces, and extremely amenable groups, Israel J. Math. 127 (2002), 317-357.

[53] D. E. Radford, A natural ring basis for the shuffle algebra and an application to group schemes, J. Algebra 58 (1979), 432-454.

[54] R. Rado, Universal graphs and universal functions, Acta Arith. 9 (1964), 331-340.

[55] C. Reutenauer, Free Lie Algebras, London Math. Soc. Monographs (New Series) 7, Oxford University Press, Oxford, 1993.

[56] F. Richman, Maximal subgroups of infinite symmetric groups, Canad. Math. Bull. 10 (1967), 375-381. 
[57] J. H. Schmerl, Countable homogeneous partially ordered sets, Algebra Universalis 9 (1979), 317-321.

[58] L. L. Scott, Representations in characteristic p, Proc. Symp. Pure Math. 37 (1980), 319-331.

[59] S. Shelah and S. Thomas, The cofinality spectrum of the infinite symmetric group, J. Symbolic Logic 62 (1997), 902-916.

[60] S. R. Thomas, Reducts of the random graph, J. Symbolic Logic 56 (1991), 176-181.

[61] S. R. Thomas, Reducts of random hypergraphs, Ann. Pure Appl. Logic 80 (1996), 165-193.

[62] J. Tits, Généralisation des groupes projectifs basée sur leurs propriétés de transitivité, Acad. Roy. Belgique Cl. Sci. Mem 27 (1952).

[63] J. Tits, Buildings of Spherical Type and Finite BN-Pairs, Lecture Notes in Math. 382, Springer-Verlag, Berlin, 1974.

[64] J. K. Truss, The group of the countable universal graph, Math. Proc. Cambridge Philos. Soc. 98 (1985), 213-245.

[65] J. K. Truss, Generic automorphisms of homogeneous structures, Proc. London Math. Soc. (3) 65 (1992), 121-141.

[66] J. K. Truss, The automorphism group of the random graph: four conjugates good, three conjugates better, Discrete Math. 268 (2003), 257-271.

[67] P. S. Urysohn, Sur un espace metrique universel, Bull. Sci. Math. 51 (1927), 1-38.

[68] W. Veech, Topological dynamics, Bull. Amer. Math. Soc. 83 (1977), 775-830.

[69] A. M. Vershik, The universal and random metric spaces, Russian Math. Surv. 356 (2004), No. 2, 65-104.

[70] F. O. Wagner, Relational structures and dimensions, pp. 153-180 in Automorphisms of First-Order Structures (ed. Kaye, R. W. and Macpherson, H. D.), Oxford University Press, Oxford, 1994.

[71] H. Wielandt, Finite Permutation Groups, Academic Press, New York, 1964.

[72] H. Wielandt, Unendliche Permutationsgruppen, Lecture Notes, Universität Tübingen, 1959. [English translation by P. Bruyns included in Wielandt's collected works: H. Wielandt, Mathematische Werke: Mathematical Works, Volume 1 (Bertram Huppert and Hans Schneider, eds.), Walter de Gruyter, Berlin, 1994.] 